\title{
Three-Wall Segment (TriSeg) Model Describing Mechanics and Hemodynamics of Ventricular Interaction
}

\author{
Joost Lumens, ${ }^{1,2}$ Tammo Delhass, ${ }^{1}$ Borut Kirn, ${ }^{2,3}$ and Theo Arts ${ }^{2}$ \\ ${ }^{1}$ Department of Physiology, Cardiovascular Research Institute Maastricht (CARIM), Maastricht University, PO Box 616, \\ 6200 MD Maastricht, The Netherlands; ${ }^{2}$ Department of Biomedical Engineering, Cardiovascular Research Institute Maastricht \\ (CARIM), Maastricht University, Maastricht, The Netherlands; and ${ }^{3}$ Institute of Physiology, School of Medicine, University \\ of Ljubljana, Ljubljana, Slovenia
}

(Received 26 November 2008; accepted 30 July 2009; published online 29 August 2009)

\begin{abstract}
A mathematical model (TriSeg model) of ventricular mechanics incorporating mechanical interaction of the left and right ventricular free walls and the interventricular septum is presented. Global left and right ventricular pump mechanics were related to representative myofiber mechanics in the three ventricular walls, satisfying the principle of conservation of energy. The walls were mechanically coupled satisfying tensile force equilibrium in the junction. Wall sizes and masses were rendered by adaptation to normalize mechanical myofiber load to physiological standard levels. The TriSeg model was implemented in the previously published lumped closed-loop CircAdapt model of heart and circulation. Simulation results of cardiac mechanics and hemodynamics during normal ventricular loading, acute pulmonary hypertension, and chronic pulmonary hypertension (including load adaptation) agreed with clinical data as obtained in healthy volunteers and pulmonary hypertension patients. In chronic pulmonary hypertension, the model predicted right ventricular free wall hypertrophy, increased systolic pulmonary flow acceleration, and increased right ventricular isovolumic contraction and relaxation times. Furthermore, septal curvature decreased linearly with its transmural pressure difference. In conclusion, the TriSeg model enables realistic simulation of ventricular mechanics including interaction between left and right ventricular pump mechanics, dynamics of septal geometry, and myofiber mechanics in the three ventricular walls.
\end{abstract}

Keywords-Pulmonary hypertension, Septal motion, Adaptation, Stress, Strain, Myofiber, Cardiac mechanics.

\section{NOMENCLATURE}

\section{General}

LV Left ventricle/ventricular

RV Right ventricle/ventricular

\begin{tabular}{|c|c|}
\hline LW & Left ventricular free wall \\
\hline SW & Septal wall \\
\hline \multirow[t]{2}{*}{ RW } & Right ventricular free wall \\
\hline & Geometry-Related Parameters \\
\hline$V_{\mathrm{LV}}$ & Left ventricular cavity volume $\left(\mathrm{m}^{3}\right)$ \\
\hline$V_{\mathrm{RV}}$ & Right ventricular cavity volume $\left(\mathrm{m}^{3}\right)$ \\
\hline$V_{\mathrm{w}}$ & Wall volume of ventricular wall segment $\left(\mathrm{m}^{3}\right)$ \\
\hline$V_{\mathrm{m}}$ & $\begin{array}{l}\text { Volume of spherical cap, formed by } \\
\text { midwall surface of wall segment }\left(\mathrm{m}^{3}\right)\end{array}$ \\
\hline$A_{\mathrm{m}}$ & $\begin{array}{l}\text { Midwall surface area of curved wall } \\
\text { segment }\left(\mathrm{m}^{2}\right)\end{array}$ \\
\hline$C_{\mathrm{m}}$ & $\begin{array}{l}\text { Curvature of midwall surface (reciprocal of } \\
\text { radius) }\left(\mathrm{m}^{-1}\right)\end{array}$ \\
\hline$x_{\mathrm{m}}$ & $\begin{array}{l}\text { Maximal axial distance from midwall sur- } \\
\text { face to origin }(\mathrm{m})\end{array}$ \\
\hline$y_{\mathrm{m}}$ & Radius of midwall junction circle (m) \\
\hline$z$ & $\begin{array}{l}\text { Ratio of wall thickness to midwall radius of } \\
\text { curvature of curved wall segment }\end{array}$ \\
\hline$\alpha$ & $\begin{array}{l}\text { Half the opening angle of spherical midwall } \\
\text { surface }\end{array}$ \\
\hline$\varepsilon_{\mathrm{f}}$ & Natural myofiber strain \\
\hline
\end{tabular}

Force-Related Parameters

$p_{\mathrm{LV}} \quad$ Left ventricular cavity pressure $(\mathrm{Pa})$

$p_{\mathrm{RV}} \quad$ Right ventricular cavity pressure $(\mathrm{Pa})$

$p_{\text {Trans }} \quad$ Transmural pressure difference across curved wall segment $(\mathrm{Pa})$

$\sigma_{\mathrm{f}} \quad$ Cauchy myofiber stress $(\mathrm{Pa})$

$T_{\mathrm{m}} \quad$ Representative midwall tension $\left(\mathrm{N} \mathrm{m}^{-1}\right)$

$T_{\mathrm{x}} \quad$ Axial midwall tension component $\left(\mathrm{N} \mathrm{m}^{-1}\right)$

$T_{\mathrm{y}} \quad$ Radial midwall tension component $\left(\mathrm{N} \mathrm{m}^{-1}\right)$

\section{INTRODUCTION}

The left (LV) and right (RV) ventricular cavities of the normal human heart are separated from the iology, Cardiovascular Research Institute Maastricht (CARIM), Maastricht University, PO Box 616, 6200 MD Maastricht, The Netherlands. Electronic mail: j.lumens@fys.unimaas.nl 

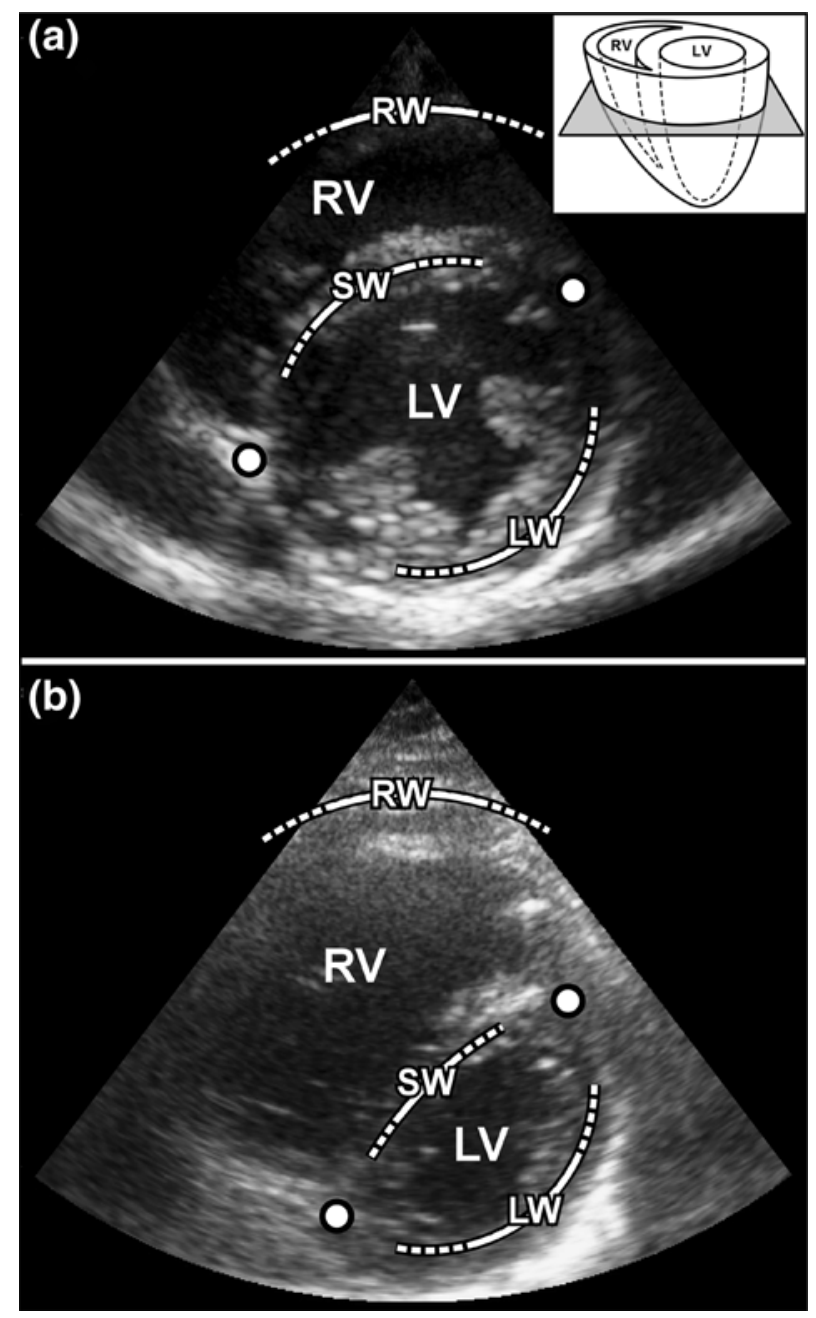

FIGURE 1. Two typical 2D echocardiographic images showing end-diastolic parasternal short-axis views of the hearts of a normal individual (a) and a pulmonary hypertension patient (b). Note that the septum is flattened with pulmonary hypertension. Cross-sections of left (LV) and right (RV) ventricular cavities, LV free wall (LW), septal wall (SW), and $R V$ free wall $(R W)$ are indicated. The bright dots indicate the cross-section of the junction margin of the walls. In the right upper corner, image plane orientation (gray plane) is indicated in a schematic representation of the cardiac ventricles.

intrathoracic space by the left and right ventricular free walls, respectively (Fig. 1a). The cavities are mutually separated by the septal wall. In order to simulate LV and RV mechanics and hemodynamics, the close anatomic coupling between these two ventricles must be considered. Experimental data demonstrate that a change in loading condition of either ventricle directly influences pump function of the other ventricle. ,21,50,55,68 $_{\text {It }}$ is also well recognized that septal geometry and motion depend on transseptal pressure difference. ${ }^{36,37,51}$ This dependency is best illustrated by flattening of the septum with increase of RV pressure relative to LV pressure (Fig. 1b). Septal geometry and motion appeared to be of diagnostic and predictive significance in patients with pulmonary hypertension. $^{32,35}$

Several mathematical models of ventricular mechanics have been developed to quantify the effect of ventricular interaction on cardiac function. In several lumped models of ventricular hemodynamics, ${ }^{7,13,41,43,48,53,56,60,61}$ ventricular interaction is described by empirically determined coupling coefficients, quantifying interventricular cross-talk of pressures and volumes. The model designed by Beyar et al. ${ }^{7}$ describes ventricular interaction in a more mechanistic way. In this model, LV and RV cavities are enclosed by three ventricular walls. For given ventricular pressures, the mechanical equilibrium of tensile forces in the junction of the walls is used as constraint to predict ventricular geometry. The model is restricted to description of passive mechanics of walls lacking contractile myofiber properties. More recently, 3D finite element models of the cardiac ventricles were used to simulate ventricular pump function and local tissue mechanics, ${ }^{34,47,66}$ inherently including ventricular interaction via the septum. In comparison with lumped models of global ventricular mechanics, finite element models allow description of regional wall mechanics and geometry. Consequently, these models are computationally demanding.

For study of beat-to-beat hemodynamics and mechanics of heart and blood vessels, Arts et al. previously developed the closed-loop CircAdapt model of heart and circulation. ${ }^{3}$ In this model, mechanical interaction of the LV and RV has been simulated by a common outer wall, having a transmural pressure equal to RV pressure, encapsulating an inner wall, which represents the left ventricle. ${ }^{1}$ The inner wall encapsulates the LV cavity only and has a transmural pressure equal to the difference between $\mathrm{LV}$ and $\mathrm{RV}$ pressure. Under normal ventricular loading conditions, this model enables realistic simulation of global LV and RV pump mechanics. ${ }^{1,3}$ However, this model setup presumes RV pressure to be substantially lower than LV pressure during the whole cardiac cycle. This condition is not satisfied with pulmonary hypertension or with left-to-right asynchrony of electrical activation. Furthermore, septal geometry cannot be simulated. Thus, this model cannot be used to interpret this measurable signal that contains important information about the difference between LV and RV pressure. ${ }^{35,51}$ Therefore, we designed the TriSeg model of ventricular mechanics that realistically incorporates ventricular interaction via the interventricular septum.

In the TriSeg model, three wall segments, i.e., the LV free wall (LW), septal wall (SW), and RV free wall (RW), meet in a junction margin, thus encapsulating the LV and RV cavities (Fig. 2a). LW and RW 

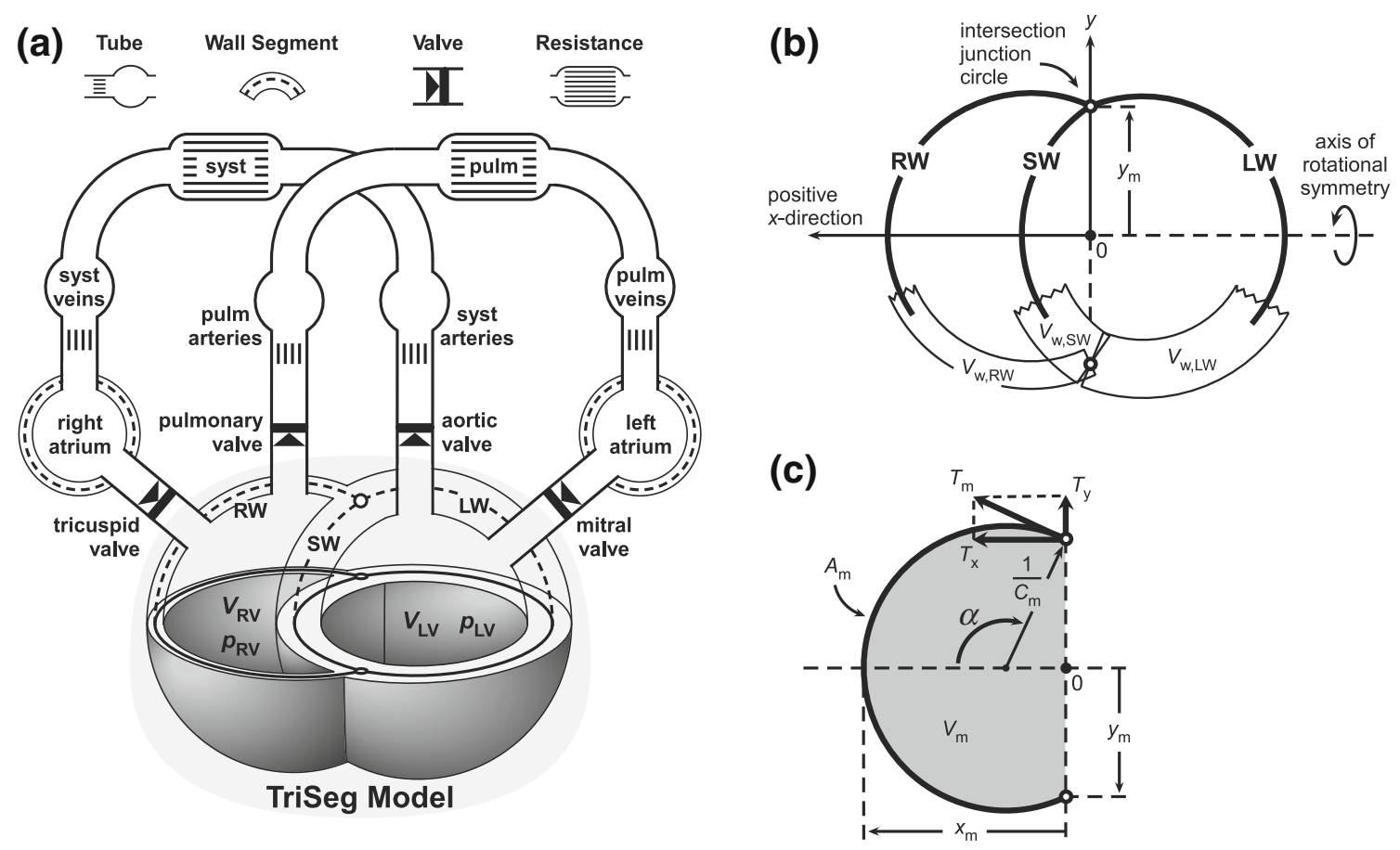

FIGURE 2. TriSeg model of ventricular mechanics. (a) The TriSeg model (gray shading) incorporated in the modular CircAdapt model of the systemic (syst) and pulmonary (pulm) circulations. ${ }^{3}$ Three thick-walled spherical segments representing LV free wall (LW), RV free wall (RW), and septal wall (SW) form a ventricular composite consisting of a left ventricular (LV) cavity, with volume $V_{\mathrm{LV}}$ and pressure $p_{\mathrm{LV}}$, and a right ventricular (RV) cavity, with volume $V_{\mathrm{RV}}$ and pressure $p_{\mathrm{RV}}$. (b) Cross-section of the ventricular composite through the axis of rotational symmetry $(x)$. The ventricular walls are coupled mechanically at the common junction circle where their midwall surfaces (thick lines) meet. This junction circle perpendicularly intersects the plane of drawing (open dots). The midwall surface divides the wall with wall volume $V_{w}$ in two shells of equal volume. The center of the junction circle is the origin ( 0 ) of the cylindrical coordinate system applied. Note that $x$-direction is defined positive toward the RV free wall. (c) Cross-section of a single wall segment through the axis of rotational symmetry. Note that only midwall geometry is shown. The shaded area indicates midwall volume $V_{m}$ enclosed between the midwall surface and the junction plane. Midwall volume $V_{m}$, area $A_{\mathrm{m}}$, and curvature $C_{\mathrm{m}}$ (reciprocal of radius of curvature) depend on distance $x_{\mathrm{m}}$ and radius $y_{\mathrm{m}}$ of the boundary circle. Each wall is loaded by transmural pressure difference resulting in a representative midwall tension $T_{m}$. With half opening angle $\alpha, T_{m}$ is resolved in an axial $\left(T_{\mathrm{x}}\right)$ and a radial $\left(T_{\mathrm{y}}\right)$ component.

geometries are calculated starting from an initial estimate of SW geometry. For each wall segment, myofiber strain is calculated from midwall surface area relative to a reference area. From myofiber strain, myofiber stress is determined using constitutive equations describing sarcomere mechanics. Substitution of myofiber stress in the known geometry of the walls results in representative midwall tension, acting on the junction line. Next, septal geometry is adjusted so that equilibrium of tension is achieved, i.e., summed tension at the junction of the three walls is equal to zero. As a result, geometries of the three wall segments and the two cavities are known, together with stresses, tensions, and pressures.

The TriSeg model of ventricular mechanics is designed to be incorporated as a module in the existing CircAdapt model simulating mechanics and hemodynamics of the whole circulation. ${ }^{3}$ According to the principles of CircAdapt, size and mass of the wall segments are determined by adaptation so that mechanical load of the myofibers is normalized to physiological standard levels. ${ }^{3,4}$ The TriSeg model, as integrated in the CircAdapt model, has been tested by simulation of time-dependent LV and RV mechanics and hemodynamics under normal ventricular loading conditions as well as with acute and chronic pulmonary hypertension $(\mathrm{PH})$. $\mathrm{PH}$ has been simulated to test whether the TriSeg model realistically relates septal geometry to transseptal pressure. Simulation results have been compared with previously published experimental data on ventricular hemodynamics ${ }^{9,62}$ and on the relation between septal geometry and transmural pressure $^{17,51}$ in healthy volunteers and in patients with chronic $\mathrm{PH}$.

\section{METHODS}

\section{General Design of the TriSeg Model}

The design of the new TriSeg model of ventricular mechanics should enable incorporation as a module in the existing CircAdapt model that simulates mechanics 
and hemodynamics of the whole circulation. ${ }^{3}$ Therefore, the TriSeg model calculates LV and RV pressures ( $p_{\mathrm{LV}}$ and $p_{\mathrm{RV}}$, respectively) as functions of $\mathrm{LV}$ and $\mathrm{RV}$ cavity volumes ( $V_{\mathrm{LV}}$ and $V_{\mathrm{RV}}$, respectively). Mechanics of ventricular interaction is incorporated assuming a simplified ventricular composite geometry (Fig. 2a). The three ventricular walls $\mathrm{LW}, \mathrm{SW}$, and RW are modeled to be thick-walled and spherical with a common junction circle with midwall radius $y_{\mathrm{m}}$ (Fig. 2b). Midwall is defined as the spherical surface that divides wall volume $V_{\mathrm{w}}$ in an inner and an outer shell of equal volume. Midwall volume $V_{\mathrm{m}}$ is the volume enclosed by the midwall surface and the plane of the junction circle (Fig. 2c). The center of the junction circle is the origin of the applied cylindrical coordinate system (Fig. 2b). The $x$-direction is perpendicular to the plane of the junction circle and is defined positive toward the RV free wall. The $y$-coordinate represents the radial distance to the $x$-axis. The spherical midwall surface of a wall segment intersects the $x$-axis at value $x_{\mathrm{m}}$ (Fig. 2c).

In presenting the TriSeg model, the following levels are distinguished: (1) ventricular hemodynamics, (2) ventricular composite mechanics, (3) ventricular wall segment mechanics, (4) curved wall patch mechanics, and (5) myofiber mechanics. Starting from LV and RV volumes, the sequence of calculations needed to obtain
$\mathrm{LV}$ and RV pressures has been illustrated in Fig. 3. The ventricular hemodynamics section serves as interface with the CircAdapt model of the whole circulation. ${ }^{3}$ In the ventricular composite section, LV and $\mathrm{RV}$ volumes are used to calculate $\mathrm{LV}$ and $\mathrm{RV}$ pressures, applying a model of wall mechanics to each ventricular wall segment. In the ventricular wall segment section, volume $V_{\mathrm{m}}$ and boundary radius $y_{\mathrm{m}}$ of a spherical wall segment are used to calculate the axial and radial vector components $T_{\mathrm{x}}$ and $T_{\mathrm{y}}$, respectively, at the common junction circle from representative midwall tension $T_{\mathrm{m}}$, applying a model of the mechanics of a curved wall patch. Such patch is defined as a fraction of a spherical wall segment with an arbitrarily shaped boundary and with midwall area $A_{\mathrm{m}}$ and curvature $C_{\mathrm{m}}$. Curvature is defined as the reciprocal of radius of curvature, having the advantage of being well defined for a flat wall. In the section of curved wall patch mechanics, $A_{\mathrm{m}}$ and $C_{\mathrm{m}}$ are used to calculate representative midwall tension $T_{\mathrm{m}}$, applying a model of myofiber mechanics. In the myofiber mechanics section, natural myofiber strain $\varepsilon_{\mathrm{f}}$ is converted to Cauchy myofiber stress $\sigma_{\mathrm{f}}$, using a constitutive law of the myofiber. This model of myofiber mechanics has been heuristically obtained from reported physiological experiments.

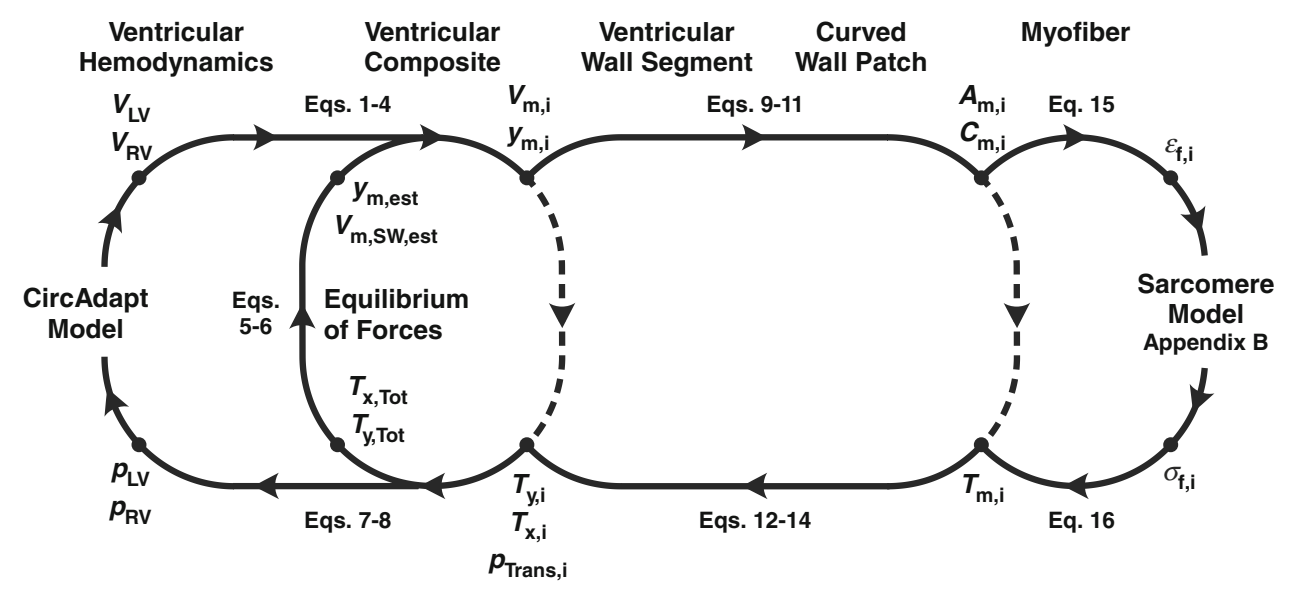

FIGURE 3. Flowchart of the TriSeg model of left (LV) and right (RV) ventricular mechanics. Applied equations are indicated by their numbers. The ventricular hemodynamics section serves as interface with the CircAdapt model of the whole circulation. In the ventricular composite section, for each wall $i$, midwall boundary radius $\left(y_{\mathrm{m}, i}\right)$ and midwall volume $\left(V_{\mathrm{m}, i}\right)$ are calculated from LV and RV volumes ( $V_{\mathrm{LV}}$ and $V_{\mathrm{RV}}$, respectively) and septal geometry. Septal geometry is initially defined by estimates of boundary radius $\left(y_{\mathrm{m}, e s t}\right)$ and midwall volume $\left(V_{\mathrm{m}, \mathrm{sw}}, \mathrm{est}\right)$. LV free wall, septum, and $\mathrm{RV}$ free wall are indicated by $i=\mathrm{LW}$, sW, and RW, respectively. The first dashed arrow indicates calculation of axial $\left(T_{x, i}\right)$ and radial $\left(T_{y, i}\right)$ wall tension components and transmural pressure $\left(p_{\text {Trans }, i}\right)$. LV and RV pressure ( $p_{\mathrm{LV}}$ and $p_{\mathrm{RV}}$, respectively) and a new estimate of septal geometry is obtained from the summed axial $\left(T_{\mathrm{x}, \mathrm{Tot}}\right)$ and radial $\left(T_{\mathrm{y}, \mathrm{Tot}}\right)$ tension components. If septal geometry is sufficiently accurate by iteration, the solution is accepted. Because direct calculation along the dashed arrow is not possible, the sequence of calculations has to follow the solid lines. For each wall segment, midwall area $\left(A_{\mathrm{m}, i}\right)$ and curvature $\left(C_{\mathrm{m}, i}\right)$ are calculated. Wall tension $\left(T_{\mathrm{m}, i}\right)$ is calculated following the second dashed arrow and renders wall tension components and transmural pressure. Again, direct calculation is not possible. Therefore, in the myofiber section, midwall area and curvature are used to calculate myofiber strain $\left(\varepsilon_{\mathrm{f}, i}\right)$. Myofiber strain is converted to myofiber stress $\left(\sigma_{\mathrm{f}, i}\right)$ using a model of sarcomere mechanics. Myofiber stress and wall segment geometry are used to calculate wall tension. By cascading the different sections, calculations can be performed sequentially along the pathways marked by solid lines. 


\section{Ventricular Composite Mechanics}

In this section, it is shown how cavity volumes $V_{\mathrm{LV}}$ and $V_{\mathrm{RV}}$ are used to calculate pressures $p_{\mathrm{LV}}$ and $p_{\mathrm{RV}}$. For that purpose, a model of ventricular wall segment mechanics is applied that renders axial and radial tension components ( $T_{\mathrm{x}}$ and $T_{\mathrm{y}}$, respectively) from representative midwall tension as a function of the midwall volume $\left(V_{\mathrm{m}}\right)$ and junction radius $\left(y_{\mathrm{m}}\right)$ of the spherical wall segment. This latter model will be derived in the next section discussing mechanics of the ventricular wall segment.

The geometry of the ventricular composite (Figs. 2b and 2c) is defined by the common radius $y_{\mathrm{m}}$ of the wall junction and the enclosed midwall cap volumes $V_{\mathrm{m}, \mathrm{LW}}$, $V_{\mathrm{m}, \mathrm{SW}}$, and $V_{\mathrm{m}, \mathrm{RW}}$ of the wall segments $\mathrm{LW}, \mathrm{SW}$, and RW, respectively. Septal midwall volume $V_{\mathrm{m}, \mathrm{SW}}$ and junction radius $y_{\mathrm{m}}$ are initially estimated by the solutions as obtained in the preceding time point, i.e., $V_{\mathrm{m}, \mathrm{SW}, \text { est }}$ and $y_{\mathrm{m}, \mathrm{sst}}$, respectively:

$$
\begin{aligned}
y_{\mathrm{m}} & =y_{\mathrm{m}, \mathrm{est}} \\
V_{\mathrm{m}, \mathrm{SW}} & =V_{\mathrm{m}, \mathrm{SW}, \text { est }}
\end{aligned}
$$

The latter estimates, together with ventricular cavity volumes $V_{\mathrm{LV}}$ and $V_{\mathrm{RV}}$ as given directly by the CircAdapt model, render an estimate of ventricular composite geometry. Using the fact that ventricular cavity volume added to half of wall volume $V_{\mathrm{w}}$ of the enclosing wall segments renders the sum of midwall volumes, it is found:

$$
\begin{aligned}
& V_{\mathrm{m}, \mathrm{LW}}=-V_{\mathrm{LV}}-\frac{1}{2} V_{\mathrm{w}, \mathrm{LW}}-\frac{1}{2} V_{\mathrm{w}, \mathrm{SW}}+V_{\mathrm{m}, \mathrm{SW}} \\
& V_{\mathrm{m}, \mathrm{RW}}=+V_{\mathrm{RV}}+\frac{1}{2} V_{\mathrm{w}, \mathrm{RW}}+\frac{1}{2} V_{\mathrm{w}, \mathrm{SW}}+V_{\mathrm{m}, \mathrm{SW}}
\end{aligned}
$$

Note that the sign of midwall volume $V_{\mathrm{m}}$ is positive if wall curvature is convex to the positive $x$-direction (Fig. 2b). Thus, for a normal heart, the sign is negative for LW and positive for RW and SW.

For each wall segment $i, V_{\mathrm{m}, i}$ and $y_{\mathrm{m}, i}$ are used to calculate axial and radial tension components $T_{\mathrm{x}, i}$ and $T_{\mathrm{y}, i}$, as well as transmural pressure $p_{\text {Trans }, i}$. This is done by applying a model of ventricular wall segment mechanics as discussed in the next section. The axial tension components of the three wall segments $T_{\mathrm{x}, \mathrm{LW}}$, $T_{\mathrm{x}, \mathrm{SW}}$, and $T_{\mathrm{x}, \mathrm{RW}}$ are summed to calculate the net axial tension $T_{\mathrm{x}, \mathrm{Tot}}$ in the junction. Similarly, the net radial tension $T_{\mathrm{y}, \mathrm{Tot}}$ in the junction is obtained. The initial estimates of $y_{\mathrm{m}}$ and $V_{\mathrm{m}, \mathrm{Sw}}$ (Eqs. 1 and 2) are adjusted numerically so that for the summed tension components it holds:

$$
T_{\mathrm{x}, \operatorname{Tot}}\left(y_{\mathrm{m}}, V_{\mathrm{m}, \mathrm{SW}}\right)=0
$$

$$
T_{\mathrm{y}, \mathrm{Tot}}\left(y_{\mathrm{m}}, V_{\mathrm{m}, \mathrm{SW}}\right)=0
$$

Having solved Eqs. (5) and (6), and assuming the pressure surrounding the ventricular composite to be zero, for LV and RV pressures it holds:

$$
\begin{aligned}
& p_{\mathrm{LV}}=-p_{\text {Trans, } \mathrm{LW}} \\
& p_{\mathrm{RV}}=+p_{\text {Trans, } \mathrm{RW}}
\end{aligned}
$$

Note that the minus sign for $p_{\mathrm{LV}}$ is caused by the definition of $p_{\text {Trans }}$, which is positive for a negative $x$-gradient of pressure in the wall segment.

\section{Mechanics of the Ventricular Wall Segment}

In this section, it is shown for a wall segment (Fig. 2c) how $V_{\mathrm{m}}$ and $y_{\mathrm{m}}$ are used to calculate axial and radial midwall tension components $T_{\mathrm{x}}$ and $T_{\mathrm{y}}$, and transmural pressure $p_{\text {Trans }}$. For that purpose, a model of the mechanics of a curved wall patch is applied. Such curved wall patch is defined as a fraction of a spherical wall segment with midwall surface area $A_{\mathrm{m}}$ and curvature $C_{\mathrm{m}}$. This latter model renders representative midwall tension from midwall surface area $A_{\mathrm{m}}$ and curvature $C_{\mathrm{m}}$ and will be derived in the next section discussing mechanics of the curved wall patch.

Wall segment geometry is most conveniently described by radius $y_{\mathrm{m}}$ of the junction circle and distance $x_{\mathrm{m}}$ between plane of junction circle and center of midwall surface (Fig. 2c). $V_{\mathrm{m}}, A_{\mathrm{m}}$, and $C_{\mathrm{m}}$ depend on $x_{\mathrm{m}}$ and $y_{\mathrm{m}}$ by

$$
\begin{gathered}
V_{\mathrm{m}}=\frac{\pi}{6} x_{\mathrm{m}}\left(x_{\mathrm{m}}^{2}+3 y_{\mathrm{m}}^{2}\right) \\
A_{\mathrm{m}}=\pi\left(x_{\mathrm{m}}^{2}+y_{\mathrm{m}}^{2}\right) \\
C_{\mathrm{m}}=\frac{2 x_{\mathrm{m}}}{\left(x_{\mathrm{m}}^{2}+y_{\mathrm{m}}^{2}\right)}
\end{gathered}
$$

First, $x_{\mathrm{m}}$ is calculated from $y_{\mathrm{m}}$ and $V_{\mathrm{m}}$ by solving Eq. (9). Second, $A_{\mathrm{m}}$ and $C_{\mathrm{m}}$ are determined with Eqs. (10) and (11). Next, $A_{\mathrm{m}}$ and $C_{\mathrm{m}}$ are used to calculate representative midwall tension $T_{\mathrm{m}}$, applying a model of the mechanics of a curved wall patch as discussed in the next section.

At the circular boundary of the midwall surface (Fig. 2c), geometric parameters $x_{\mathrm{m}}$ and $y_{\mathrm{m}}$, and midwall tension $T_{\mathrm{m}}$ are used to calculate axial $\left(T_{\mathrm{x}}\right)$ and radial $\left(T_{\mathrm{y}}\right)$ tension components:

$$
\begin{gathered}
T_{\mathrm{x}}=T_{\mathrm{m}} \cdot \sin \alpha \quad \text { with } \sin \alpha=\frac{2 x_{\mathrm{m}} y_{\mathrm{m}}}{x_{\mathrm{m}}^{2}+y_{\mathrm{m}}^{2}} \\
T_{\mathrm{y}}=T_{\mathrm{m}} \cdot \cos \alpha \quad \text { with } \cos \alpha=\frac{-x_{\mathrm{m}}^{2}+y_{\mathrm{m}}^{2}}{x_{\mathrm{m}}^{2}+y_{\mathrm{m}}^{2}}
\end{gathered}
$$


The symbol $\alpha$ represents half opening angle of the circular wall segment (Fig. 2c).

Transmural pressure $p_{\text {Trans }}$ equals total axial force. Consequently, $p_{\text {Trans }}$ is calculated by multiplying $T_{\mathrm{x}}$ by the ratio of length of the junction contour $\left(2 \pi y_{\mathrm{m}}\right)$ to area of the junction circle $\left(\pi y_{\mathrm{m}}^{2}\right)$ :

$$
p_{\text {Trans }}=\frac{2 T_{\mathrm{x}}}{y_{\mathrm{m}}}
$$

\section{Mechanics of the Curved Wall Patch}

In this section, it is shown how midwall surface area $A_{\mathrm{m}}$ and curvature $C_{\mathrm{m}}$ are used to calculate representative midwall tension $T_{\mathrm{m}}$. For that purpose, a constitutive model of myofiber mechanics is applied. This latter model renders Cauchy myofiber stress from natural myofiber strain and is presented in Appendix B.

In the ventricular module, originally implemented in the CircAdapt model, ${ }^{3}$ the one-fiber model developed by Arts et $a l^{2}{ }^{2}$ was used to relate ventricular pump mechanics, as described by cavity pressure and volume, to myofiber mechanics, as described by myofiber stress and strain. When assembling three wall segments to a ventricular composite with two cavities, contractile function of a wall segment, as described by representative midwall tension and area change, should be put between mechanics of cavity and myofiber. The following conditions should be satisfied. First, when folding a wall segment to a completely closed spherical surface, the relation between pump mechanics and myofiber mechanics should be equivalent to the equations of the one-fiber model. Second, like in the one-fiber model, where contractile myofiber work equals ventricular pump work, summed pump work of both cavities should be equal to summed work as generated by the three walls. Furthermore, within each wall segment, work as delivered by the wall through wall tension and changes of geometry, should be equal to the work generated by the myofibers.

In Appendix A, it is shown that such a model of wall segment mechanics can be found, although the derivation is quite complex. Because the shape of the midwall area is not relevant for the balance of work, the relations are extended to the more general case of a spherical wall patch with arbitrary shape of the boundary. Thus, for a wall patch it is found that natural myofiber strain $\varepsilon_{\mathrm{f}}$ depends on midwall surface area $A_{\mathrm{m}}$ and curvature $C_{\mathrm{m}}$ by the following approximation (relative error $<1 \%$ ), as derived in Appendix A:

$$
\varepsilon_{\mathrm{f}} \approx \frac{1}{2} \ln \left(\frac{A_{\mathrm{m}}}{A_{\mathrm{m}, \mathrm{ref}}}\right)-\frac{1}{12} z^{2}-0.019 z^{4} \quad \text { with } \quad z=\frac{3 C_{\mathrm{m}} V_{\mathrm{w}}}{2 A_{\mathrm{m}}}
$$

where $A_{\mathrm{m}, \text { ref }}$ represents reference midwall surface area. Note that the dimensionless curvature parameter $z$ is closely related to the dimensionless ratio of wall thickness to radius of curvature. With a constitutive model of the myofiber (Appendix B), natural myofiber strain $\varepsilon_{\mathrm{f}}$ is used to calculate Cauchy myofiber stress $\sigma_{\mathrm{f}}$. In Appendix A, it is derived how myofiber stress and wall segment geometry are used to calculate representative midwall tension $T_{\mathrm{m}}$. It holds by approximation (relative error $<2 \%$ ):

$$
T_{\mathrm{m}} \approx \frac{V_{\mathrm{w}} \sigma_{\mathrm{f}}}{2 A_{\mathrm{m}}}\left(1+\frac{z^{2}}{3}+\frac{z^{4}}{5}\right) \quad \text { with } \quad \sigma_{\mathrm{f}}=f\left(\varepsilon_{\mathrm{f}}\right)
$$

Equations (15) and (16) represent the first few terms of a Taylor series approximating the analytically derived expressions. These approximations have the advantage of being well defined for zero curvature. The analytical derivations cannot be used in numerical calculations, because zero by zero division occurs near zero curvature, while the ratio is analytically well defined.

\section{Implementation of TriSeg Model in CircAdapt Model}

The TriSeg model of ventricular mechanics is incorporated in the existing CircAdapt model of the whole circulation. ${ }^{3}$ This latter model supplies the required hemodynamic boundary conditions, i.e., LV and RV cavity volumes. The CircAdapt model is designed as a network of modules representing cardiac chambers, valves, large blood vessels, and peripheral resistances. Extended with the TriSeg model, the CircAdapt model allows beat-to-beat simulation of time-dependent ventricular mechanics and hemodynamics, e.g., ventricular cavity volumes and pressures, geometries and representative myofiber mechanics of ventricular walls, and flows through valves. An important feature of the existing CircAdapt model is that the number of independent parameters is reduced by incorporating adaptation of cavity size and wall mass of cardiac chambers and blood vessels to mechanical load so that stresses and strains in the walls of heart and blood vessels are normalized to tissuespecific physiological standard levels.

The model was initialized using realistic estimates of wall volume $V_{\mathrm{w}}$, reference midwall surface area $A_{\mathrm{m} \text {,ref, }}$ and reference sarcomere length $L_{\mathrm{s}, \text { ref }}$ (Table 1: Parameter values for model initialization). After model initialization, these parameters will be obtained for each wall segment by load adaptation as described in the "Simulations" section. The actual parameter values chosen for initialization (Table 1) are noncritical due to load adaptation. However, for fast convergence to steady-state adaptation, these estimates were chosen in physiological range. 
TABLE 1. Input parameter values for NORM and PH simulations.

\begin{tabular}{|c|c|c|}
\hline Parameter & Unit & Value \\
\hline \multicolumn{3}{|l|}{ Hemodynamics at rest } \\
\hline Mean systemic arterial blood pressure & $\mathrm{kPa}$ & 12.2 \\
\hline Mean systemic blood flow & $m L s^{-1}$ & 85 \\
\hline Cardiac cycle time & $\mathrm{s}$ & 0.850 \\
\hline $\begin{array}{l}\text { Mean pulmonary arteriovenous pressure drop } \\
\text { Interventions with exercise }\end{array}$ & $\mathrm{kPa}$ & 1.5 (NORM) \\
\hline Mean systemic blood flow & $\mathrm{mL} \mathrm{s} \mathrm{s}^{-1}$ & 255 \\
\hline Cardiac cycle time & $\mathrm{s}$ & 0.425 \\
\hline \multicolumn{3}{|l|}{ Set point values for adaptation } \\
\hline Mean flow velocity in large blood vessels (rest) & $\mathrm{m} \mathrm{s}^{-1}$ & 0.17 \\
\hline Maximum vascular wall stress (exercise) & $\mathrm{kPa}$ & 500 \\
\hline Maximum sarcomere length (exercise) & $\mu \mathrm{m}$ & 2.2 \\
\hline Minimum sarcomere length (exercise) & $\mu \mathrm{m}$ & 1.75 \\
\hline $\begin{array}{l}\text { Maximum passive myofiber stress (exercise) } \\
\text { Interventions with PH simulations }\end{array}$ & Interventions with $\mathrm{PH}$ simulations & 10 (LW), 8 (SW), 20 (RW) \\
\hline Mean pulmonary arteriovenous pressure drop & $\mathrm{kPa}$ & $\begin{array}{l}3.0 \text { (PHAc1 and PHCh1), } \\
4.5 \text { (PHAc2 and PHCh2), } \\
6.0 \text { (PHAc3 and PHCh3) }\end{array}$ \\
\hline \multicolumn{3}{|l|}{ Parameter values for model initialization } \\
\hline Wall volume $\left(V_{\mathrm{w}}\right)$ & $\mathrm{mL}$ & 75 (LW), 40 (SW), 30 (RW) \\
\hline Reference midwall surface area $\left(A_{\mathrm{m}, \mathrm{ref}}\right)$ & $\mathrm{cm}^{2}$ & 80 (LW), 45 (SW), 100 (RW) \\
\hline Reference sarcomere length $\left(L_{s, \text { ref }}\right)$ & $\mu \mathrm{m}$ & $2.0(\mathrm{LW}, \mathrm{SW}$, and $\mathrm{RW})$ \\
\hline Septal midwall volume $\left(V_{\mathrm{m}, \mathrm{sw}}\right)$ & $\mathrm{mL}$ & 42 \\
\hline Radius of midwall junction circle $\left(y_{\mathrm{m}}\right)$ & $\mathrm{cm}$ & 3.3 \\
\hline
\end{tabular}

LW, left ventricular free wall; NORM, normal simulation; PHAc, acute pulmonary hypertension simulation; $\mathrm{PHCh}$, chronic pulmonary hypertension simulation; RW, right ventricular free wall; SW, septal wall.

The set of differential equations that describe the CircAdapt model, including the TriSeg module, was solved by numerical integration with time steps of $2 \mathrm{~ms}$ using the ODE113 function in MATLAB 7.1.0 (MathWorks, Natick, MA). Simulation time of a single cardiac cycle was less than $7 \mathrm{~s}$ on a Windows $\mathrm{XP}^{\mathrm{TM}}$ platform (version 2002) with a $2.00 \mathrm{GHz}$ Intel ${ }^{\circledR}$ Core $^{\mathrm{TM}} 2$ Duo T7250 processor and 1 GB of RAM.

\section{Simulations}

The TriSeg model, as integrated in the CircAdapt model, was tested by simulation of human ventricular mechanics and hemodynamics with (1) normal ventricular loading conditions (NORM), (2) acute pulmonary hypertension (PHAc), and (3) chronic pulmonary hypertension including adaptation $(\mathrm{PHCh})$. All simulations are presented with similar values of mean systemic blood flow (cardiac output), cardiac cycle time (heart rate), and mean systemic arterial blood pressure, simulating hemodynamics at rest (Table 1: Hemodynamics at rest).

\section{NORM Simulation}

The diameter of large blood vessels was assumed to be determined by adaptation to mean chronic circumstances, which state is most closely described by the condition of rest (Table 1: Hemodynamics at rest). However, geometry of the heart and wall thickness of large blood vessels were considered to be a result of adaptation to a state of moderate exercise, thus simulating the effect of relatively short periods of training by exercise. The applied simulation protocol of adaptation has been described earlier. ${ }^{3}$ Briefly recapitulating, with resting hemodynamics (Table 1: Hemodynamics at rest), diameters of the large blood vessels were adapted until mean blood flow velocity reached the set point value (Table 1: Set point values for adaptation). Next, a moderate state of exercise was simulated by tripling cardiac output and doubling heart rate (Table 1: Interventions with exercise). Under these circumstances, wall volume $\left(V_{\mathrm{w}}\right)$ and reference midwall surface area $\left(A_{\mathrm{m}, \mathrm{ref}}\right)$ of all cardiac wall segments were adapted until maximum and minimum sarcomere length as well as maximum passive myofiber stress reached the set point values of adaptation with exercise (Table 1). Also, wall thickness of the blood vessels was adapted until maximum wall stress was equal to the set point value (Table 1). Next, hemodynamics were returned to rest conditions and the abovementioned adaptation protocol was repeated two or three times until steady-state geometry was reached ( $<1 \%$ deviation from set point values). In total, finding the steady-state adapted NORM simulation required simulation of about 100-200 cardiac cycles. 


\section{PHAc and PHCh Simulations}

The NORM simulation was used as point of departure for simulation of ventricular mechanics and hemodynamics with increased pulmonary resistance. Steady-state simulations (PHAc1, PHAc2, and PHAc3), representing increasing degrees of acute pulmonary hypertension, were obtained by acute increase of mean pulmonary arteriovenous pressure drop in three steps without adaptation (Table 1: Interventions with $\mathrm{PH}$ simulations). Finally, adaptation was applied to the three steady-state PHAc simulations following the same protocol used for the NORM simulation. This resulted in three steady-state simulations (PHCh1, $\mathrm{PHCh} 2$, and $\mathrm{PHCh} 3$, respectively), representing gradually increasing degrees of chronic pulmonary hypertension at rest.

\section{Simulation Data Analysis}

\section{Ventricular Pump Mechanics and Hemodynamics}

Simulated time courses of normal (NORM) LV and $\mathrm{RV}$ pressures, volumes, and flows were compared with physiological data obtained in normal subjects. ${ }^{26,29,54,62,63}$ Area of ventricular pressure-volume relation was calculated to quantify ventricular pump stroke work ( $\left.W_{\text {stroke }}\right)$ for the LV and RV. Time courses of blood flow velocities through the mitral, aortic, tricuspid, and pulmonary valves were used to study the effects of chronic pulmonary hypertension on ventricular hemodynamics. For comparison with clinical data obtained in healthy subjects and in patients with chronic pulmonary hypertension, ${ }^{9,62}$ several timing parameters of RV hemodynamics were quantified for the NORM and PHCh simulations. RV ejection time (ET) was quantified as the time from pulmonary valve opening to closure, acceleration time of pulmonary flow (AT) as the time from pulmonary valve opening to moment of maximal pulmonary flow velocity, RV isovolumic contraction time (ICT) as the time from tricuspid valve closure to pulmonary valve opening, and $\mathrm{RV}$ isovolumic relaxation time (IRT) as the time from pulmonary valve closure to tricuspid valve opening.

\section{Tissue Mechanics}

Area of myofiber stress-strain relation was calculated to quantify stroke work density ( $\left.w_{\text {stroke }}\right)$ for the LW, SW, and RW. Stroke work density was defined as contractile myofiber stroke work per unit of tissue volume.

\section{Ventricular Wall Geometry}

End-diastolic wall thicknesses $\left(H_{\text {ed }}\right)$ were calculated as wall volume divided by end-diastolic midwall surface area. End-diastole was defined as the moment of mitral valve closure. Furthermore, time courses of LW, SW, and RW midwall curvatures were used to assess acute and chronic effects of increase of pulmonary resistance on wall curvatures. The relation between septal geometry and the transseptal pressure gradient in the TriSeg model was compared with the relation found in patients with and without chronic pulmonary hypertension. ${ }^{17}$ For that purpose, curvature ratio (CR) was calculated as the $\mathrm{SW} / \mathrm{LW}$ midwall curvature ratio at the moment of aortic valve closure, whereas transmural pressure ratio (PR) was defined as the difference between maximum LV and RV pressures divided by maximum LV pressure.

\section{RESULTS}

\section{Simulation of Normal Physiology (NORM)}

Figure 4 shows simulated time courses of ventricular cavity pressures and volumes, aortic and pulmonary artery pressures, and flows through arterial and atrioventricular valves under normal ventricular loading conditions (NORM). These time courses show agreement with physiological data on the following aspects: (1) LV and RV cavity pressures and volumes rise at the end of diastole as a result of atrial contraction, (2) time courses of mitral and tricuspid valve flows show an early passive filling wave (E) followed by a clearly separated late filling wave (A) resulting from atrial contraction, ${ }^{26}$ (3) LV and RV end-diastolic volumes are not significantly different, ${ }^{54}$ (4) RV ejection starts earlier and ends later than LV ejection, ${ }^{29}$ and (5) durations of $\mathrm{LV}$ isovolumic contraction and relaxation exceed the corresponding RV time intervals (Fig. 4, bottom panel). ${ }^{62,63}$ Steady-state adaptation rendered LW, SW, and RW wall volumes of 76,38 , and $31 \mathrm{~mL}$, respectively. Wall thicknesses $H_{\text {ed,Lw }}$ and $H_{\text {ed,Sw }}$ were almost equal and three times as large as $H_{\mathrm{ed}, \mathrm{RW}}$ (Table 2).

\section{Simulations of Acute Pulmonary Hypertension (PHAc)}

The upper panel row of Fig. 5 illustrates simulated ventricular pressure-volume loops under normal loading conditions (NORM) and with acutely increased pulmonary resistance (PHAc1, PHAc2, and PHAc3). With increasing pulmonary resistance, RV cavity volume, systolic and diastolic RV pressures, mean pulmonary artery pressure, and RV pump work (Table 3) increased, while LV cavity volume and pump work were relatively unaffected. The middle panel row of Fig. 5 shows stress-strain loops of the myofibers in the ventricular wall segments. Acute rise of pulmonary resistance caused increase of RW myofiber stress, myofiber strain, and stroke work density, while it 

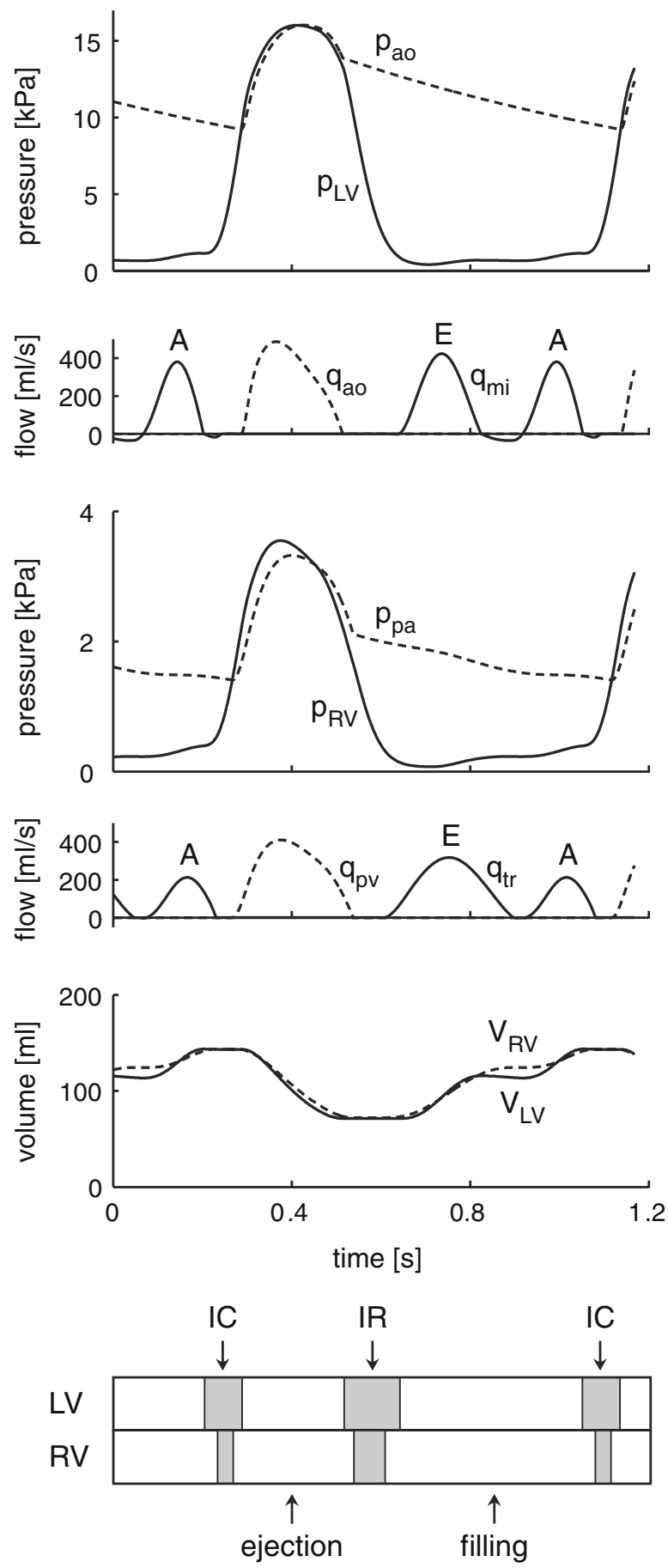

FIGURE 4. Simulated time courses of normal (NORM) LV and RV hemodynamics at rest. The bar plot (bottom panel) displays RV and LV ejection, filling, and isovolumic contraction and relaxation periods. Note that simulation duration amounts to one and a half cardiac cycle. Symbols: A, late active ventricular filling wave; $E$, early passive ventricular filling wave; IC, isovolumic contraction; IR, isovolumic relaxation; LV, left ventricular; $p_{\mathrm{ao}}$, aortic pressure; $p_{\mathrm{LV}}$, left ventricular pressure; $p_{\mathrm{pa}}$, pulmonary artery pressure; $p_{\mathrm{Rv}}$, right ventricular pressure; $q_{\mathrm{aoo}}$, aortic valve flow; $q_{\mathrm{mi}}$, mitral valve flow; $q_{\mathrm{pv}}$, pulmonary valve flow; $q_{\mathrm{tr}}$, tricuspid valve flow; RV, right ventricular; $V_{\mathrm{LV}}$, left ventricular cavity volume; and $V_{\mathrm{RV}}$, right ventricular cavity volume.
TABLE 2. Simulation results: ventricular wall thickness.

\begin{tabular}{|c|c|c|c|c|c|c|c|c|}
\hline \multirow[b]{2}{*}{ Parameter } & \multirow[b]{2}{*}{ Unit } & \multirow[b]{2}{*}{ NORM } & \multicolumn{3}{|c|}{ PHAc } & \multicolumn{3}{|c|}{$\mathrm{PHCh}$} \\
\hline & & & 1 & 2 & 3 & 1 & 2 & 3 \\
\hline \multicolumn{9}{|c|}{ End-diastolic wall thickness } \\
\hline$H_{\mathrm{ed}, \mathrm{LW}}$ & $\mathrm{mm}$ & 7.9 & 7.8 & 7.6 & 7.3 & 8.3 & 8.2 & 8.3 \\
\hline$H_{\text {ed,sw }}$ & $\mathrm{mm}$ & 6.8 & 6.9 & 7.0 & 6.9 & 6.7 & 6.9 & 6.5 \\
\hline$H_{\mathrm{ed}, \mathrm{RW}}$ & $\mathrm{mm}$ & 2.3 & 2.1 & 2.0 & 1.7 & 3.3 & 4.2 & 5.1 \\
\hline
\end{tabular}

$H_{\text {ed }}$, end-diastolic wall thickness; LV, left ventricular; LW, left ventricular free wall; NORM, normal simulation; PHAc, acute pulmonary hypertension simulation; $\mathrm{PHCh}$, chronic pulmonary hypertension simulation; RV, right ventricular; RW, right ventricular free wall; SW, septal wall.

caused decrease of LW and SW stroke work densities (Table 3). The lower panel row of Fig. 5 illustrates time courses of $\mathrm{LW}, \mathrm{SW}$, and RW midwall curvatures. With increasing pulmonary resistance, SW midwall curvature decreased predominantly during diastole. In the PHAc3 simulation, the septum even flattened to zero curvature and bulged shortly toward the LV free wall (negative curvature) during early diastole. LW and RW curvatures appeared relatively insensitive to acute changes of pulmonary resistance. With increasing pulmonary resistance (Table 2 ), $H_{\text {ed,Rw }}$ decreased maximally by $26 \%$ in PHAc3 relative to NORM, $H_{\text {ed,LW }}$ decreased maximally by $8 \%$, whereas $H_{\text {ed,SW }}$ increased only $1 \%$.

\section{Simulations of Chronic Pulmonary Hypertension ( $\mathrm{PHCh}$ )}

Figure 6 illustrates graphs of the same variables as presented in Fig. 5, but now rendered after adaptation to increased pulmonary resistance (PHCh1, PHCh2, and PHCh3). Like in absence of adaptation, systolic and diastolic RV pressures, mean pulmonary artery pressure, and RV pump work were elevated with chronic pulmonary hypertension (Table 3), while LV pump function remained relatively unaffected. In contrast to the PHAc simulations, RV cavity volume decreased $8 \%$ in the PHCh3 simulation (Fig. 6). The myofiber stress-strain loops in Fig. 6 show that adaptation resulted in a more homogeneous distribution of stroke work density in the ventricular walls (Table 3). In the PHCh3 simulation, RW stroke work density was increased $(20 \%)$, whereas LW and SW stroke work densities were decreased (7 and 5\%, respectively) relative to the NORM simulation. Increase of pulmonary resistance with load adaptation resulted in the following changes of ventricular geometry. Mean SW curvature (Fig. 6) decreased linearly with increasing pulmonary resistance, while the shape of the time course was preserved. LW and RW 

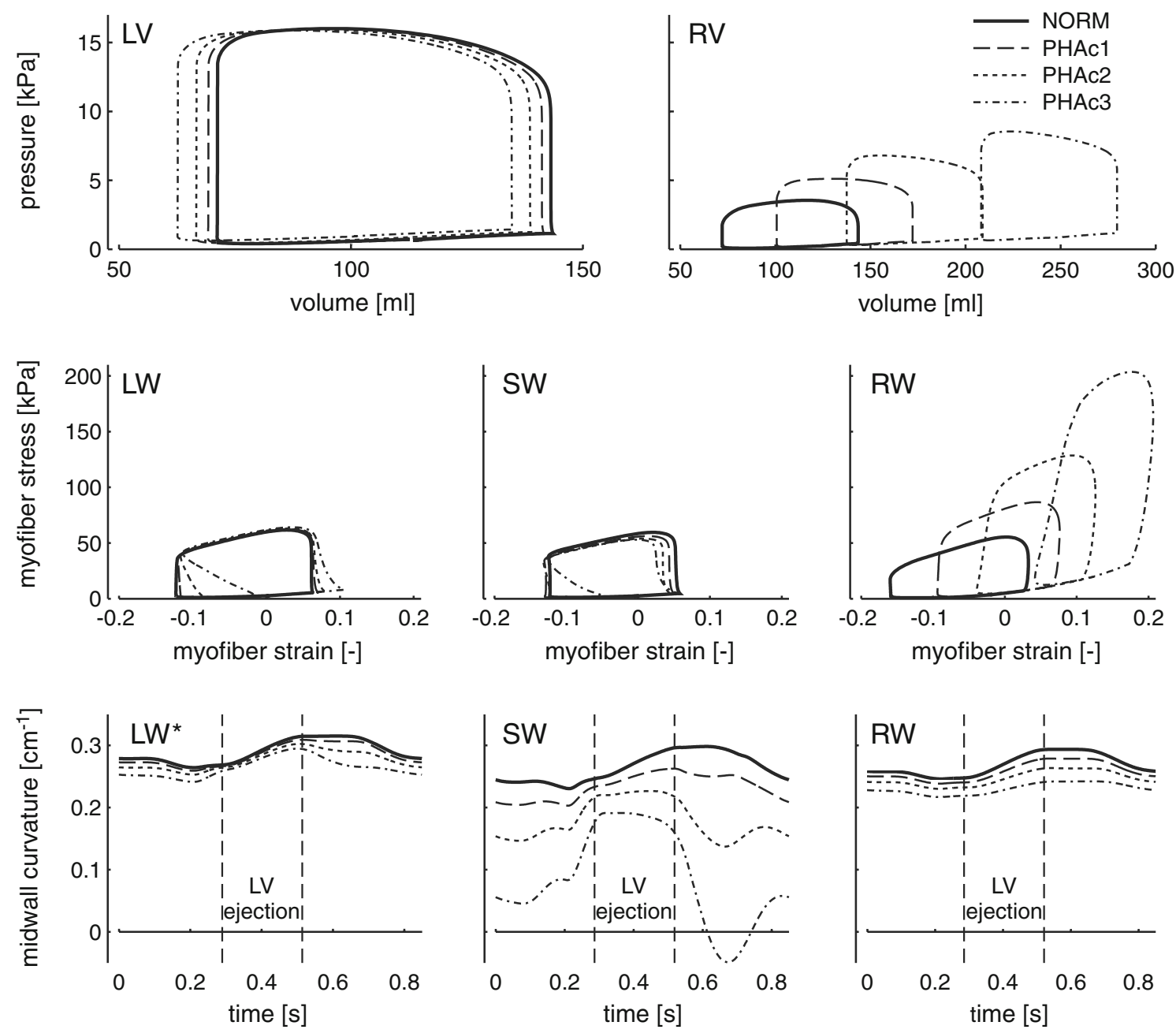

FIGURE 5. Simulated ventricular pressure-volume loops, myofiber stress-strain loops, and time courses of midwall curvatures under normal (NORM) and acute pulmonary hypertensive (PHAc1, PHAc2, and PHAc3) ventricular loading conditions. Acute increase of pulmonary resistance resulted in RV dilatation, increased RV pump work, increased contractile work density in the RV free wall, and decreased septal midwall curvature. Simulation duration amounts to one cardiac cycle. LV ejection is indicated in the curvature plots. Abbreviations: LV, left ventricular; LW, left ventricular free wall; RV, right ventricular; RW, right ventricular free wall; and SW, septal wall. *Note that the absolute value of LW midwall curvature is used for graphical representation, while this curvature is defined to be negative in the TriSeg model.

TABLE 3. Simulation results: pulmonary artery pressures and ventricular work.

\begin{tabular}{|c|c|c|c|c|c|c|c|c|}
\hline \multirow[b]{2}{*}{ Parameter } & \multirow[b]{2}{*}{ Unit } & \multirow[b]{2}{*}{ NORM } & \multicolumn{3}{|c|}{ PHAc } & \multicolumn{3}{|c|}{$\mathrm{PHCh}$} \\
\hline & & & 1 & 2 & 3 & 1 & 2 & 3 \\
\hline \multicolumn{9}{|c|}{ Ventricular pump stroke work } \\
\hline$W_{\text {stroke, LV }}$ & $\mathrm{Pa} \mathrm{m}{ }^{3}$ & 1.04 & 1.04 & 1.03 & 1.02 & 1.05 & 1.04 & 1.04 \\
\hline $\begin{array}{l}\text { Wtroke,RV } \\
\text { Stroke work }\end{array}$ & $\begin{array}{l}\mathrm{Pa} \mathrm{m}^{3} \\
\text { ity }\end{array}$ & \multicolumn{7}{|c|}{ Stroke work density } \\
\hline$w_{\text {stroke }, \mathrm{LW}}$ & $\mathrm{kPa}$ & 9.41 & 9.54 & 9.32 & 8.66 & 8.88 & 8.90 & 8.78 \\
\hline$W_{\text {stroke, } \mathrm{SW}}$ & $\mathrm{kPa}$ & 8.94 & 8.28 & 7.54 & 6.15 & 9.19 & 8.66 & 8.49 \\
\hline$W_{\text {stroke, } \mathrm{RW}}$ & $\mathrm{kPa}$ & 7.91 & 11.66 & 15.94 & 21.59 & 8.59 & 9.13 & 9.48 \\
\hline \multicolumn{9}{|c|}{ Mean pulmonary artery pressure } \\
\hline$p_{\mathrm{pa}}$ & $\mathrm{kPa}$ & 2.04 & 3.56 & 5.11 & 6.77 & 3.53 & 5.00 & 6.47 \\
\hline
\end{tabular}

LV, left ventricular; LW, left ventricular free wall; NORM, normal simulation; PHAc, acute pulmonary hypertension simulation; $\mathrm{PHCh}$, chronic pulmonary hypertension simulation; $p_{\mathrm{pa}}$, mean pulmonary artery pressure; RV, right ventricular; RW, right ventricular free wall; SW, septal wall; $w_{\text {stroke, }}$ stroke work density; $W_{\text {stroke }}$, ventricular pump stroke work. 

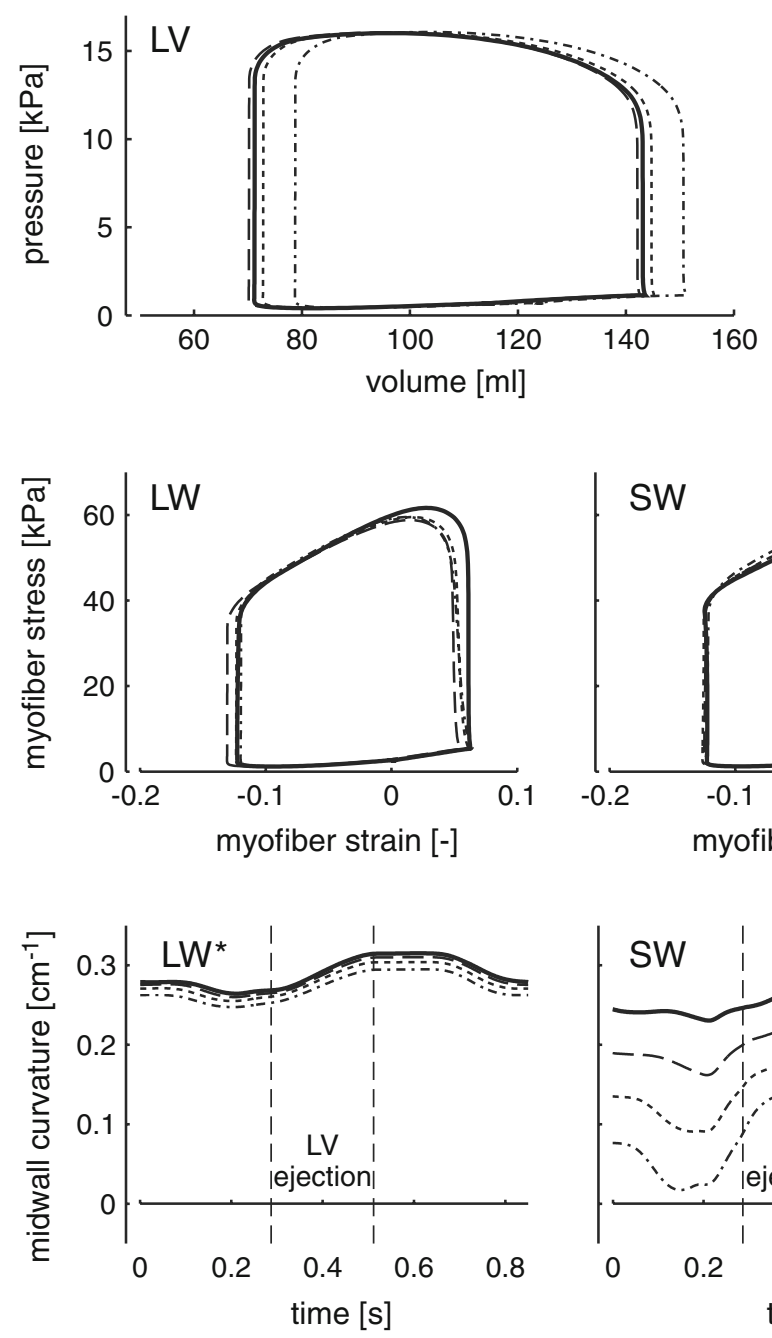
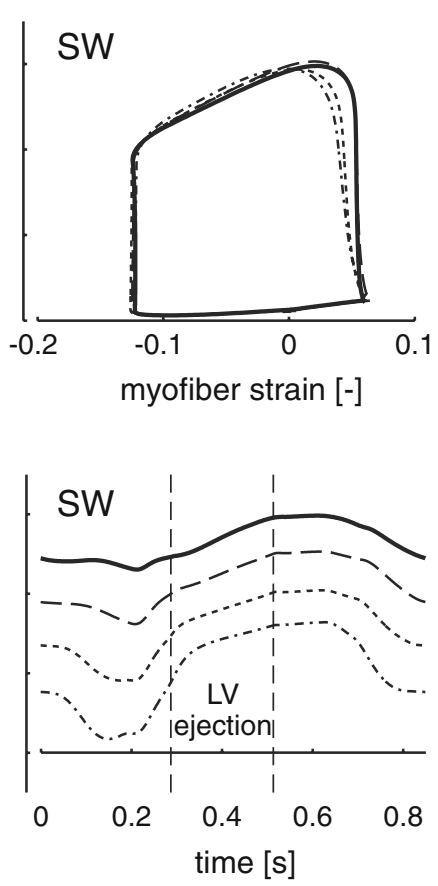
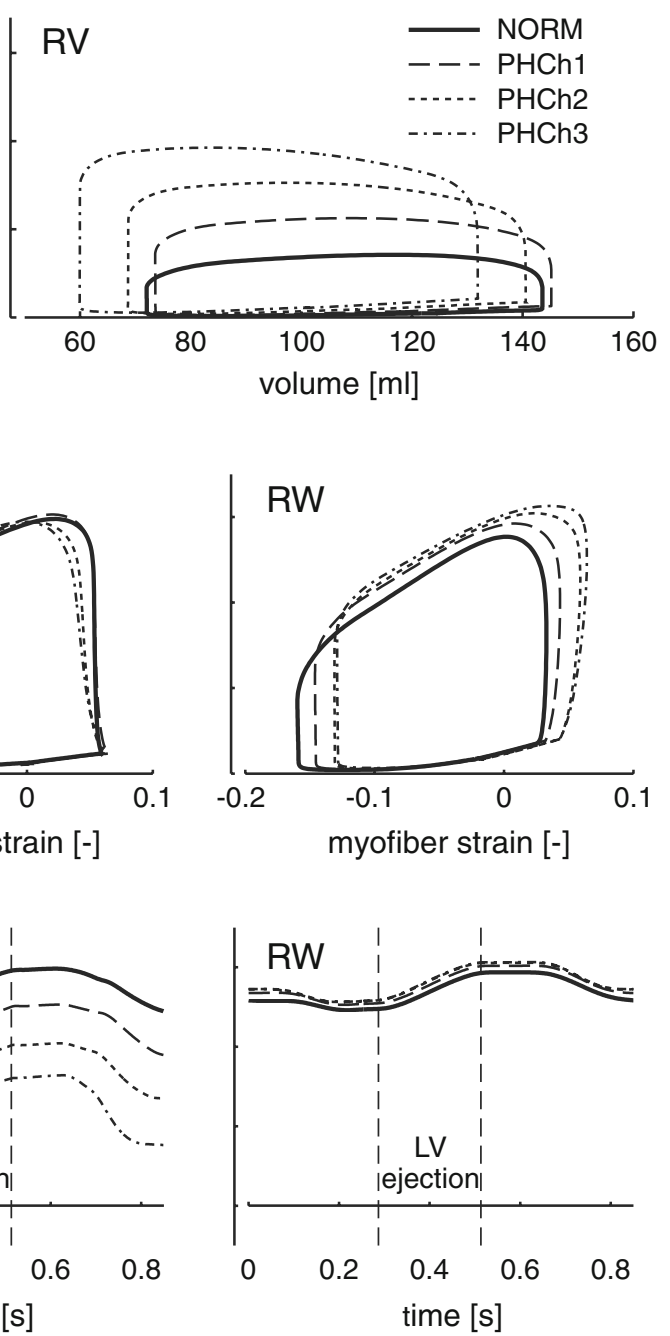

FIGURE 6. Simulated ventricular pressure-volume loops, myofiber stress-strain loops, and time courses of midwall curvatures under normal (NORM) and chronic pulmonary hypertensive (PHCh1, PHCh2, and PHCh3) ventricular loading conditions. Chronic increase of pulmonary resistance including adaptation resulted in increase of RV pump work, homogeneity of contractile work densities in the walls, and linear decrease of septal midwall curvature. Simulation duration amounts to one cardiac cycle. LV ejection is indicated in the curvature plots. Abbreviations: LV, left ventricular; LW, left ventricular free wall; RV, right ventricular; RW, right ventricular free wall; and SW, septal wall. *Note that the absolute value of LW midwall curvature is used for graphical representation, while this curvature is defined to be negative in the TriSeg model.

curvatures were practically unaffected. Relative to the NORM simulation, $H_{\text {ed,RW }}$ increased up to $120 \%$ in PHCh3, whereas changes of $H_{\text {ed,Lw }}$ and $H_{\text {ed,Sw }}$ were small $(<5 \%)$ (Table 2$)$.

In Fig. 7, time courses of LV and RV inflow and outflow velocities are shown for the NORM and PHCh simulations. With increasing pulmonary resistance, pulmonary and tricuspid valve flow velocities changed significantly relative to the NORM simulation, whereas aortic and mitral valve flow velocities remained relatively unaffected. Furthermore, in Fig. 7, RV ejection time (ET), acceleration time of pulmonary flow (AT), RV isovolumic contraction time (ICT), and $\mathrm{RV}$ isovolumic relaxation time (IRT) are indicated for the NORM simulation. In Fig. 8, changes of these timing parameters are compared with the changes as observed in clinical studies among normal individuals and patients with chronic pulmonary hypertension. ${ }^{9,62}$ In the simulations, chronic increase of pulmonary resistance caused decrease of AT and AT/ET-ratio, indicating increase of pulmonary flow curve skewness. This is in accordance with measurements as obtained in patients with chronic pulmonary hypertension. ${ }^{9}$ Furthermore, simulated ICT and IRT lengthened (maximally $163 \%$ and $47 \%$ in the $\mathrm{PHCh} 3$ simulation, respectively). The ratio of total $\mathrm{RV}$ isovolumic time $($ ICT + IRT) to ET almost doubled. Again, the simulated changes were similar to the changes as observed in patients with pulmonary hypertension. ${ }^{62}$ Furthermore, the tricuspid early filling wave narrowed and the 

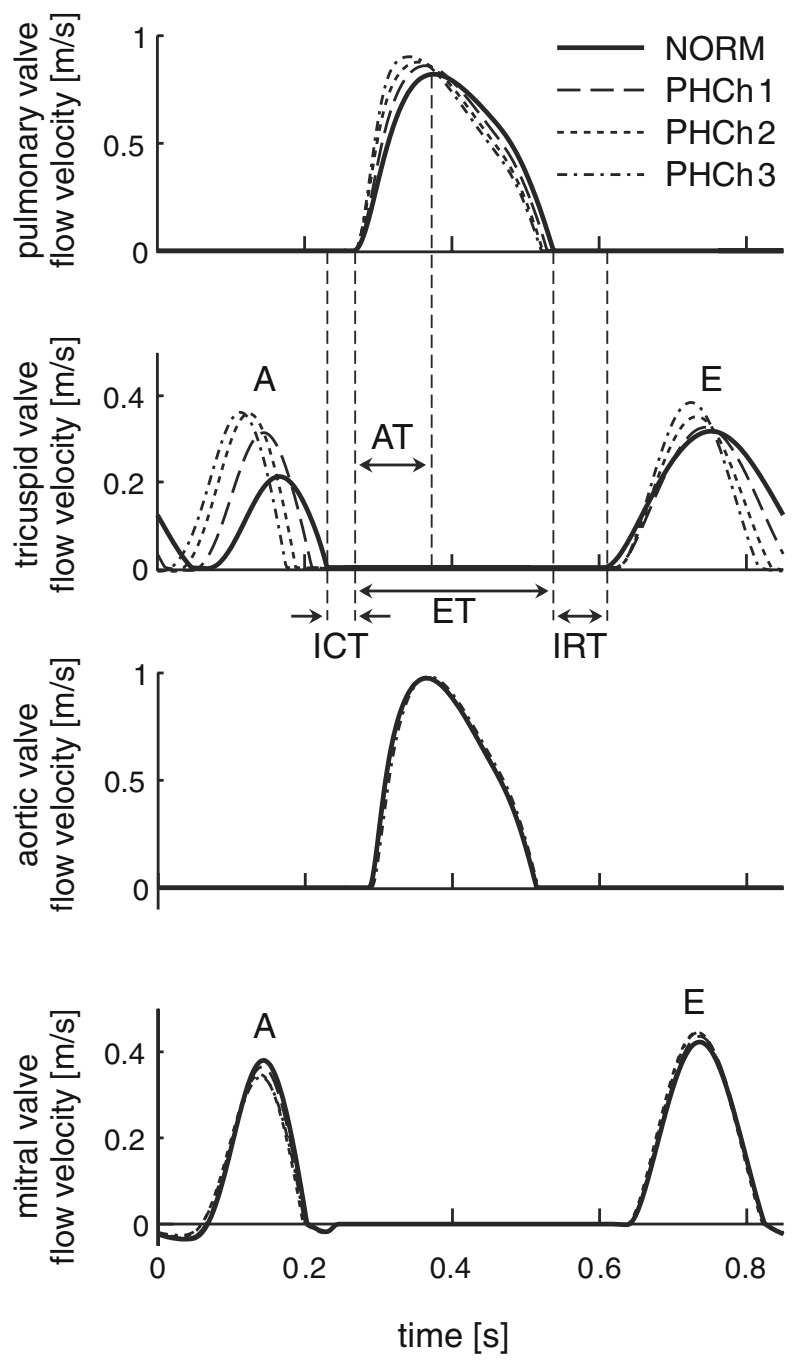

FIGURE 7. Simulated time courses of pulmonary and tricuspid valve flow velocities (upper two panels) show lengthening of RV isovolumic contraction, shortening of RV ejection, and increase of pulmonary flow acceleration after adaptation to increased pulmonary resistance (PHCh1, PHCh2, and PHCh3). Aortic and mitral valve flow velocities (lower two panels) appear relatively insensitive to increase of pulmonary resistance. Simulation duration amounts to one cardiac cycle. The following time intervals are indicated for the NORM simulation: AT, acceleration time of pulmonary valve flow; ET, RV ejection time; ICT, RV isovolumic contraction time; and IRT, RV isovolumic relaxation time. Symbols: A, late active ventricular filling wave; and E, early passive ventricular filling wave.

tricuspid E/A-ratio of peak atrioventricular flow during early filling $(\mathrm{E})$ to that during atrial contraction (A) decreased from 1.49 in the NORM simulation to unity in the PHCh3 simulation.

Figure 9 shows simulated time courses of SW/LW transmural pressure ratio and $\mathrm{SW} / \mathrm{LW}$ midwall curvature ratio. SW transmural pressure was quantified as LV cavity pressure minus RV cavity pressure, while LW transmural pressure was quantified as LV cavity pressure. At each time point in the cardiac cycle, both ratios decreased linearly with increase of pulmonary resistance.

Both CR and PR decreased with increase of pulmonary resistance (Table 4). Figure 10 shows the relation between $\mathrm{CR}$ and $\mathrm{PR}$, as derived from the NORM and PHCh simulations, as well as the relation found among patients with and without pulmonary hypertension. ${ }^{17}$ In this latter patient population, pulmonary hypertension was defined as an RV systolic pressure $>40 \mathrm{mmHg}$. Linear regression analysis of data points as derived from the NORM and PHCh simulations revealed a strong correlation $\left(r^{2}=1.00\right.$, SEE $=0.008)$ between CR and PR with similar slope as the relation found in patients $\left(r^{2}=0.73, \mathrm{SEE}=0.044\right)$. In the simulations, however, an offset was found, shifting the relation to higher CR values (Fig. 10). It is noted (not shown) that although acute change $( \pm 20 \%)$ of mean systemic flow or mean arterial blood pressure affected CR and PR, their interdependence followed the linear relation as derived from the chronic simulations within $2 \%$ deviation. The same holds for the data points derived from the PHAc1 and PHAc2 simulations, while for the PHAc3 simulation the deviation was more than 10\% (Table 4, not shown in Fig. 10).

\section{DISCUSSION}

The newly designed TriSeg model of ventricular mechanics incorporates mechanical interaction of the LV free wall, RV free wall, and septal wall resulting in a strong coupling of LV and RV pump mechanics and hemodynamics. The TriSeg model was successfully implemented as a module in the available CircAdapt model, simulating cardiac mechanics and hemodynamics of the closed-loop circulation. Effects of ventricular interaction on cardiac mechanics and hemodynamics were assessed by simulation of pulmonary hypertension in the acute phase as well as in the chronic phase, the latter implying adaptation of ventricular geometry to mechanical load. For chronic pulmonary hypertension, simulated ventricular geometry, hemodynamics, and septal mechanics agreed surprisingly well with corresponding measurements in patients.

\section{Model Assumptions}

In the TriSeg model, ventricular geometry was approximated by three thick-walled spherical segments encapsulating the LV and RV cavities (Fig. 2a). In reality, the ventricular cavities are enclosed by truncated ellipsoidal muscular walls and the noncontractile basal sheet with valves. ${ }^{58}$ The simplification to spherical segments without a noncontractile sheet resulted in 

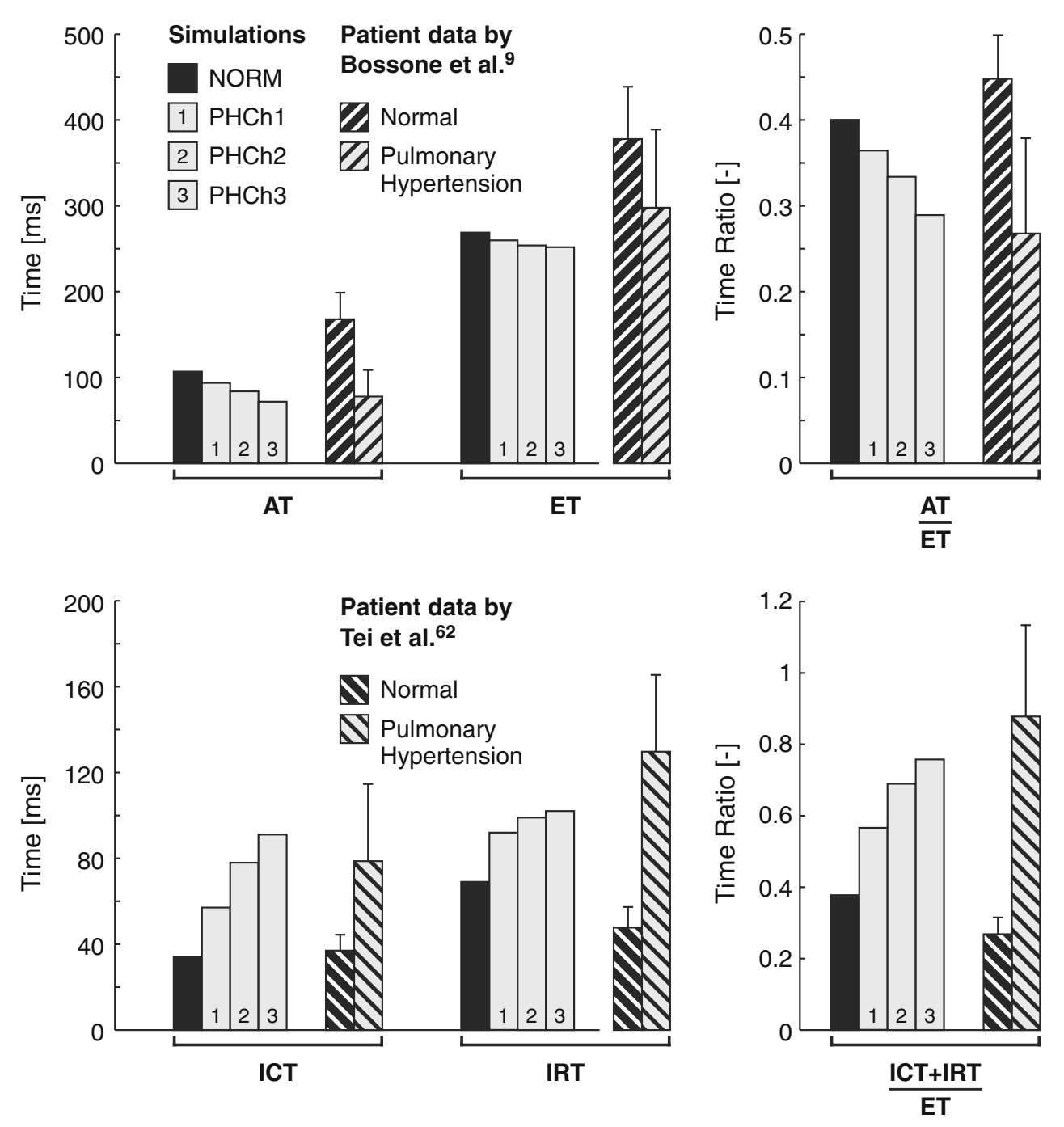

FIGURE 8. Comparison of simulated and patient data on timing of RV hemodynamics. Simulated changes of AT, ET, ICT, and IRT as chronic response to increased pulmonary resistance agree with measurements obtained in healthy normals and patients with pulmonary hypertension [adapted from Bossone et al. ${ }^{9}$ (upper row) and Tei et al. ${ }^{62}$ (lower row)]. Patient data are presented by mean values with standard deviations indicated by error bars. Symbols: AT, pulmonary flow acceleration time; ET, RV ejection time; ICT, RV isovolumic contraction time; and IRT, RV isovolumic relaxation time.

a general underestimation of ventricular dimensions. Although the fact that there are indications that bending stiffness of the myocardium may be important for simulation of septal geometry, ${ }^{7,21}$ we neglected this effect for simplicity. Despite these inaccuracies, relative changes of dimensions during the cardiac cycle as a result of adaptation were simulated realistically.

In the TriSeg model, myofiber strain was estimated from midwall curvature, area, and wall volume by application of the one-fiber model ${ }^{2}$ to a spherical wall segment (Eq. 15 and Appendix A). The one-fiber model has been shown to be insensitive to actual wall geometry by assuming conservation of energy and homogeneity of fiber stress in the wall. ${ }^{2}$ So, we expected that the present relation for transmural pressure as a function of midwall surface area and curvature was also applicable to the real, more irregular cardiac geometry, although this fact has not been proven.

The analytically derived dependency of myofiber strain on wall segment geometry (Eq. A7) appeared continuous and differentiable around $z=0$. To avoid numerical inaccuracy near zero curvature, because of zero-division, a fourth-order Taylor series approximation (Eq. 15) was used instead. For similar reasons, Eq. (A4) for midwall tension was also approximated by a fourth-order Taylor series (Eq. 16). Within the physiological range of ventricular geometry, the errors of the approximations as compared to the analytically derived relations were smaller than $1 \%$ and $2 \%$ for strain and tension, respectively. With respect to total ventricular pump work, total myofiber stroke work 


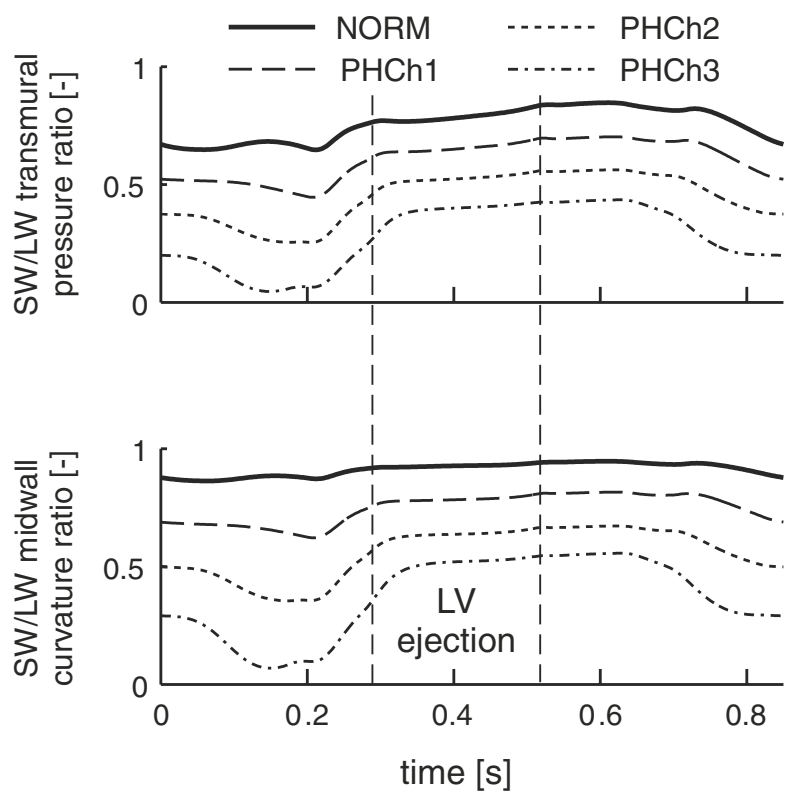

FIGURE 9. Simulated time courses of SW/LW transmural pressure ratio (upper panel) and SW/LW midwall curvature ratio (lower panel) appear similar revealing a linear relation between both ratios at each time point in the cardiac cycle. Simulation duration amounts to one cardiac cycle. Duration of LV ejection is indicated by vertical dashed lines.

TABLE 4. Simulation results: curvature ratio and transmural pressure ratio.

\begin{tabular}{|c|c|c|c|c|c|c|c|c|}
\hline \multirow[b]{2}{*}{ Parameter } & \multirow[b]{2}{*}{ Unit } & \multirow[b]{2}{*}{ NORM } & \multicolumn{3}{|c|}{ PHAc } & \multicolumn{3}{|c|}{$\mathrm{PHCh}$} \\
\hline & & & 1 & 2 & 3 & 1 & 2 & 3 \\
\hline CR & - & 0.94 & 0.85 & 0.72 & 0.54 & 0.81 & 0.67 & 0.55 \\
\hline PR & - & 0.78 & 0.68 & 0.57 & 0.46 & 0.65 & 0.52 & 0.40 \\
\hline
\end{tabular}

CR, septal-to-LV free wall midwall curvature ratio at the moment of aortic valve closure; NORM, normal simulation; PHAc, acute pulmonary hypertension simulation; $\mathrm{PHCh}$, chronic pulmonary hypertension simulation; PR, systolic septal-to-LV free wall transmural pressure ratio.

was overestimated less than $2.6 \%$, whereas total tensile stroke work at the midwall surface was underestimated less than $0.5 \%$.

In the NORM and PHCh simulations, a load-controlling adaptation mechanism was applied to render size and mass of each wall segment. The applied adaptation rules required prescribed values for maximum and minimum sarcomere lengths and for maximum passive myofiber stress in each wall segment (Table 1). Maximum and minimum sarcomere lengths were derived from experiments on isolated cardiac muscle of the rat. ${ }^{15,31,64}$ Maximum passive myofiber stress was chosen as adaptation stimulus because experimental data obtained in dogs with chronic volume overload suggested that end-diastolic myofiber stress and ejection strain were important mechanical

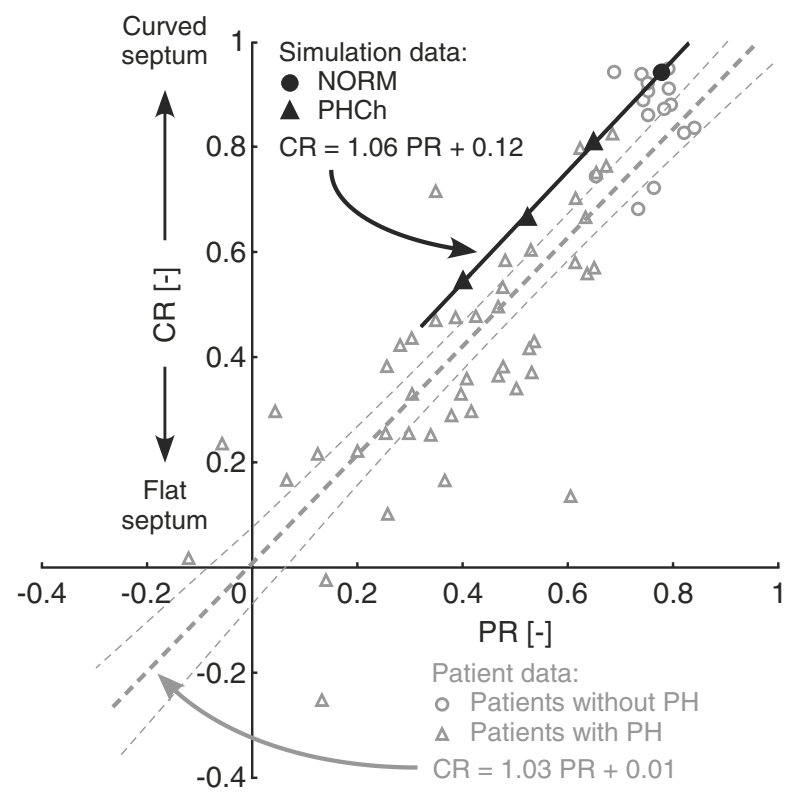

FIGURE 10. Simulation results on the correlation between curvature ratio $C R$ and transmural pressure ratio PR (black data points), see text for definitions, as compared with the relation extracted from data (gray data points) obtained in patients with and without pulmonary hypertension ( $\mathrm{PH}=\mathrm{RV}$ systolic pressure $>\mathbf{4 0} \mathrm{mmHg}$ ) [adapted from Dellegrottaglie et al. ${ }^{17}$ ]. Linear regression analysis of data points as derived from the NORM and PHCh simulations revealed a strong correlation (bold black line, $r^{2}=1.00$, SEE $=0.008$ ) between CR and PR with similar slope as the relation found in patients (thick gray dashed line, $r^{2}=0.73$, SEE $\left.=0.044\right)$. The thin dashed lines indicate the $95 \%$ confidence interval of linear regression fit to the patient data. Linear regression formulas are shown for simulated as well as measured data points. Symbols: $r$, regression residual and SEE, standard error of the estimate.

stimuli for hypertrophy, while peak systolic myofiber stress appeared irrelevant. ${ }^{22}$ However, when assuming similar values of maximum passive myofiber stress in the three ventricular walls of the TriSeg model, ventricular geometry did not develop anatomically accurate. Therefore, in our model, we adjusted levels of maximum diastolic stress per wall segment (Table 1) so that after adaptation the weight ratios for the walls agreed with findings in healthy volunteers. ${ }^{19,28}$

In this study, the external pressure surrounding the $\mathrm{LV}$ and RV free walls was assumed to be zero. The real heart is surrounded by the pericardium, which constrains increase of total heart volume during volume overload. Under resting conditions, the pericardium is believed to play a minor role, setting pericardial pressure close to zero. ${ }^{42,46,67}$ However, with acute increase of total heart volume, the pericardium affects cardiac hemodynamics and interaction of the cardiac chambers significantly. ${ }^{6,11,25}$ In our simulations of acute pulmonary hypertension (PHAc), the effect of the pericardium should be considered because of severe RV dilatation (Fig. 5). In the PHAc3 simulation, RV cavity volume even increased by $120 \%$ relative to the 
NORM simulation. Together with the changes of right atrial and LV cavity volumes ( $45 \%$ increase and $10 \%$ decrease, respectively), total heart volume increased by $25 \%$. In the chronic PH simulations, the effect of the pericardium on ventricular mechanics is likely to be of minor importance, because measurements demonstrated adaptive dilatation of the pericardium in patients with chronic pulmonary hypertension. ${ }^{8,24}$

\section{Comparison of Model Simulations with Measurements}

The relation between curvature ratio $\mathrm{CR}$ and transmural pressure ratio $\mathrm{PR}$ as extracted from the NORM and PHCh simulations (Fig. 10) agreed quite well with the relation as found in a patient group consisting of patients with and without chronic pulmonary hypertension. ${ }^{17}$ The relations were about linear with equal slope. In the simulations, however, an offset was found, shifting the relation to higher CR values, implying overestimation of septal curvature for a given right-to-left pressure ratio.

The overestimation of septal curvature may have many causes. In the model, wall geometry was considered spherical having a clear unique radius of curvature by definition. The real ventricular walls are not spherical, ${ }^{58}$ implying that radii of curvature along the circumferential and base-to-apex direction are different. Also, the junction of the real ventricular walls is smoothed over the wall boundaries, thus further hindering a clear definition of curvature. Besides an error in the curvature ratio as derived from the simulations, the offset might originate from a systematic error in the pressure or curvature measurements obtained in patients as discussed by Dellegrottaglie et al. ${ }^{17}$ Nonetheless, the linear relationship between CR and PR might be a useful tool for noninvasive estimation of systolic $\mathrm{RV}$ cavity pressure in patients with $\mathrm{RV}$ pressure overload. ${ }^{17,35,51}$ In the simulations, the linear relation appeared also valid ( $<2 \%$ deviation) for the PHAc1 and PHAc2 simulations (Table 4 ) as well as after acute changes $( \pm 20 \%)$ of mean arterial pressure and cardiac output in the chronic pulmonary hypertension simulations (not shown in Fig. 10). The latter findings suggested that the linear relation between $C R$ and $P R$ is insensitive to acute changes of hemodynamic status.

Experimental studies with acute manipulation of transseptal pressure difference as well as clinical studies among patients with chronic pulmonary hypertension showed that septal curvature and position of the septum between the LV and RV free walls depend instantaneously on transseptal pressure difference. ${ }^{10,20,21,35,36,52}$ This dependency was found during systole as well as diastole. Similar dependencies were found in our simulations of pulmonary hypertension. Septal curvature decreased with increase of pulmonary resistance (Figs. 5 and 6). Furthermore, leftward shift of the septum, increase of RV volume, and decrease of LV volume with acute increase of pulmonary resistance (Fig. 5), as predicted by the TriSeg model, were also predicted by Kerckhoffs et al. ${ }^{34}$ using a finite element model of the ventricles coupled to a lumped circulation model.

Simulations and clinical observations were in agreement concerning changes in timing of cardiac flow events due to pulmonary hypertension (Figs. 7 and 8). For example, acceleration time of pulmonary flow velocity was significantly decreased and varied linearly with mean pulmonary artery pressure. ${ }^{9,14}$ Furthermore, $\mathrm{RV}$ isovolumic contraction and relaxation times increased while pulmonary ejection time decreased. ${ }^{62,70}$ Moreover, tricuspid E/A-ratio was decreased in patients with chronic pulmonary hypertension indicating deterioration of RV diastolic function. ${ }^{71}$

Ventricular wall volumes and end-diastolic wall thicknesses (Table 2) in the NORM simulation were all about $25 \%$ smaller than values measured in healthy volunteers. ${ }^{19,28,57}$ This difference most likely resulted from an overestimation of contractility used in the sarcomere mechanics model (Appendix B, Eq. B7), causing walls to be thinner after adaptation to mechanical load. Furthermore, pulmonary acceleration and ejection times as derived from the NORM simulation (Fig. 8) were underestimated as compared to data obtained in healthy individuals. ${ }^{9,27}$ A probable cause is inaccuracy in the model description of sarcomere mechanics, which is primarily derived from experiments on isolated cardiac muscle of the rat. ${ }^{15,31,64}$ Human myocardium under in vivo conditions is likely to behave differently.

In the PHCh simulations, LV pump function and hemodynamics were relatively unaffected by increase of pulmonary resistance (Figs. 6 and 7). Also in rats with chronic pulmonary hypertension, resting $\mathrm{LV}$ pump function remains unaffected as long as the myocardium is able to compensate for increased tissue load by structural adaptation. ${ }^{23}$ Furthermore, in the PHCh simulations, the RV free wall hypertrophied and RV cavity volume decreased (Table 2 and Fig. 6). These geometric changes were in agreement with experimental observations in rats with mild chronic pulmonary hypertension. ${ }^{49}$ In the latter study, chronic pulmonary artery banding resulted in an increase of RV systolic pressure from 33 to $71 \mathrm{mmHg}$. This increase of $\mathrm{RV}$ afterload resulted in $76 \%$ increase of thickness of the RV free wall and $14 \%$ decrease of RV free wall area, which suggests a small reduction in $\mathrm{RV}$ cavity volume. In our simulations, RV systolic pressure increased from $27 \mathrm{mmHg}$ in the NORM simulation to $72 \mathrm{mmHg}$ in the PHCh3 simulation (Fig. 6), end-diastolic wall thickness increased $120 \%$ (Table 2), and RV 
cavity volume decreased $8 \%$. In another animal study in rats, ${ }^{44}$ it was shown that structural adaptation to chronic increase of RV afterload was associated with concentric hypertrophy up to a certain level. Beyond this level, however, the myocardium could not fully compensate for further load increase. Consequently, the RV cavity dilated, mainly due to dilatation of the RV free wall. In patients with severe chronic pulmonary hypertension, RV free wall hypertrophy is found to occur together with RV dilatation. ${ }^{12,30}$ Secondary to RV failure, systolic and diastolic LV function deteriorate by ventricular interaction. ${ }^{38,39}$ As a result, cardiac performance deteriorates and exercise capacity reduces. ${ }^{12,45}$ These phenomena indicating RV failure were also found in our PHAc simulations.

In the PHAc simulations, the myocardial tissue was unable to compensate for increased RV afterload by load adaptation. As a result, mechanical myofiber load was inhomogeneously distributed over the ventricular walls (Table 3 and Fig. 5). RW stroke work density increased with increase of RV afterload, whereas LW and SW stroke work densities decreased. In the PHAc3 simulation, RW stroke work density was increased by almost $200 \%$ with respect to the NORM simulation. When assuming stroke work density to be correlated to oxygen consumption, ${ }^{59}$ this implies an increase of oxygen demand by the RV free wall. Since oxygen supply is not included in our model, the potential effect of perfusion imposed limitations on myocardial performance is unknown in our simulations of pulmonary hypertension.

In patients with pulmonary hypertension, tricuspid regurgitation often occurs due to $\mathrm{RV}$ and tricuspid annular dilatation. ${ }^{18,65}$ In our simulations, tricuspid valve regurgitation was not included. In the PHAc3 simulation, RV end-diastolic volume was increased by almost $100 \%$ with respect to the NORM simulation. It is likely that the absence of tricuspid regurgitation resulted in underestimation of $\mathrm{RV}$ volume overload in the PHAc simulations.

The TriSeg model was successfully integrated as a module in the CircAdapt model of the closed-loop cardiovascular system. As shown previously, the CircAdapt environment is flexible by its modular setup and enables realistic simulation of cardiovascular mechanics and hemodynamics under normal as well as various pathological conditions. ${ }^{3,33,40}$ A set of physiological adaptation rules, expressing structural adaptation of the system to mechanical load, makes the model self-structuring and reduces the number of independent model parameters. For each ventricular wall segment in the TriSeg model, wall volume, midwall area, and reference sarcomere length were varied so that mechanical myofiber load was normalized to a known physiological level, which was assumed to be the same for all ventricular walls. The field of application of the CircAdapt model was substantially enlarged by implementation of the TriSeg model. In the future, the combined model may be used to study fundamental research questions concerning ventricular interaction and its role in cardiac pathologies. For example, specific material properties may be changed per wall segment in order to study effects of heterogeneity of wall properties on cardiac mechanics and hemodynamics, e.g., asynchronous mechanical activation (and pacing) or a localized myocardial infarct. ${ }^{33,40}$

Although the TriSeg model can be easily modified to include a description of inhomogeneous myocardial wall properties, a finite element model of cardiac mechanics is more accurate and better suited to describe local inhomogeneities in mechanical load. For patient-specific modeling of the circulation, the model should simulate many cycles in order to find a best match with the available set of measurements. For that purpose, the TriSeg model is to be preferred because calculation effort was about 1,000 times less than that of a finite element approach. The finite element model is absolutely needed to estimate and evaluate possible errors introduced by the applied simplifications of the TriSeg model. Currently, the CircAdapt model with the TriSeg module will be evaluated in its possibility to simulate hemodynamics of pulmonary hypertension patient specifically.

\section{CONCLUSIONS}

We presented the TriSeg model of ventricular mechanics and hemodynamics incorporating mechanical interaction of the LV free wall, RV free wall, and septal wall, resulting in a strong coupling of LV and RV hemodynamics. The model enables calculation of LV and RV pressures given the respective cavity volumes. LV and RV hemodynamics are related to myofiber mechanics in the three ventricular walls, satisfying the principle of conservation of energy. The three ventricular walls are mechanically coupled satisfying equilibrium of tensile forces in their junction. After implementation as a module in the lumped closed-loop CircAdapt model of heart and circulation, the TriSeg model enables simulation of ventricular hemodynamics and wall mechanics as functions of time.

Simulations of cardiac mechanics and hemodynamics during normal ventricular loading, acute pulmonary hypertension, and chronic pulmonary hypertension after load adaptation agreed with clinical data as obtained in normal subjects and in chronic pulmonary hypertension patients. With increasing levels of chronic pulmonary hypertension, the TriSeg model predicted increase of systolic pulmonary flow acceleration, increase of isovolumic contraction and 
relaxation times, and linear decrease of septal-to-LV free wall curvature ratio. Summarizing, the TriSeg model realistically describes ventricular mechanics including the interaction between left and right ventricular pump mechanics, dynamics of septal geometry, and contractile myofiber function in the three ventricular walls.

\section{APPENDIX A: DERIVATIONS OF MECHANICS OF THE CURVED WALL PATCH}

This appendix contains the analytical derivations of Eqs. (15) and (16) relating mechanics of the curved wall patch to myofiber mechanics. For the derivation of Eq. (16), calculating representative midwall tension $T_{\mathrm{m}}$ from myofiber stress, the logarithmic one-fiber model relating global ventricular pump mechanics to myofiber mechanics is used. ${ }^{2}$ Applying the definition of midwall surface as used in Eqs. (3) and (4), for the ratio of transmural pressure $p_{\text {Trans }}$ to myofiber stress $\sigma_{\mathrm{f}}$ in a closed thick-walled sphere with midwall volume $V_{\mathrm{m}}$ and wall volume $V_{\mathrm{w}}$, it holds:

$$
\frac{p_{\text {Trans }}}{\sigma_{\mathrm{f}}}=\left.\frac{1}{3} \ln \left(\frac{1+z}{1-z}\right)\right|_{\text {sphere }} \text { with } z=\left.\frac{V_{\mathrm{w}}}{2 V_{\mathrm{m}}}\right|_{\text {sphere }}
$$

where the definition of $z$ is equivalent to the more general definition used in Eq. (15) for a curved wall patch. For a closed sphere with midwall surface area $A_{\mathrm{m}}$ and curvature $C_{\mathrm{m}}$, the relation between $p_{\text {Trans }}$ and representative midwall tension $T_{\mathrm{m}}$ is determined by conservation of energy, i.e.,

$$
p_{\text {Trans }} d V_{\mathrm{m}}=\left.T_{\mathrm{m}} d A_{\mathrm{m}}\right|_{\text {sphere }}
$$

where $d V_{\mathrm{m}}$ and $d A_{\mathrm{m}}$ represent small increments of midwall volume and area, respectively. Substitution of $V_{\mathrm{m}}=$ (4/3) $\pi r^{3}, A_{\mathrm{m}}=4 \pi r^{2}$, and $r=1 / C_{\mathrm{m}}$ in Eq. (A2) yields:

$$
p_{\text {Trans }}=2 T_{\mathrm{m}} C_{\mathrm{m}}
$$

Note that Eq. (A3) represents Laplace's law expressed in such a way that the applied scalar definition of $T_{\mathrm{m}}$ satisfies conservation of energy (Eq. A2). Although we have derived Eq. (A3) for a complete sphere, it also holds for a fraction of the same sphere, since both curvature $C_{\mathrm{m}}$ and tension $T_{\mathrm{m}}$ are local parameters. Thus, Eq. (A3) may also be applied to a spherical wall segment (Fig. 2c). Substituting Eq. (A3) in Eq. (A1) and applying $C_{\mathrm{m}}=\left(2 A_{\mathrm{m}} z\right) /\left(3 V_{\mathrm{w}}\right)$ renders for representative midwall tension $T_{\mathrm{m}}$ in a curved wall patch:

$$
T_{\mathrm{m}}=\frac{\sigma_{\mathrm{f}} V_{\mathrm{w}}}{2 A_{\mathrm{m}}} \cdot \frac{1}{2 z} \ln \left(\frac{1+z}{1-z}\right)
$$

Note that this latter function is continuous around zero curvature. To avoid numerical inaccuracy near $z=0$, Eq. (A4) is replaced by its close fourth-order polynomial approximation as specified in Eq. (16) (relative error $<2 \%$ ).

For the derivation of Eq. (15), myofiber strain $\varepsilon_{\mathrm{f}}$ is considered a function of two variables, i.e., $A_{\mathrm{m}}$ and $C_{\mathrm{m}}$. Applying the principle of conservation of energy, work of the wall by a small area change $d A_{\mathrm{m}}$ with tension $T_{\mathrm{m}}$ equals work of the myofibers by a change of natural myofiber strain $d \varepsilon_{\mathrm{f}}$ with Cauchy myofiber stress $\sigma_{\mathrm{f}}$ :

$$
T_{\mathrm{m}} d A_{\mathrm{m}}=V_{\mathrm{w}} \sigma_{\mathrm{f}} d \varepsilon_{\mathrm{f}}
$$

Rearranging Eq. (A5), substituting $T_{\mathrm{m}}$ with Eq. (A4), and keeping $C_{\mathrm{m}}$ constant renders the following partial derivative:

$$
\frac{\partial \varepsilon_{\mathrm{f}}}{\partial A_{\mathrm{m}}}=\frac{1}{4 A_{\mathrm{m}} z} \ln \left(\frac{1+z}{1-z}\right) \quad \text { with } z=\frac{3 C_{\mathrm{m}} V_{\mathrm{w}}}{2 A_{\mathrm{m}}}
$$

A standard mathematical solution technique is used to find the general solution of the first-order partial differential equation in Eq. (A6). ${ }^{16}$ For that purpose, $\varepsilon_{\mathrm{f}}$ is written as the sum of a strain term $\varepsilon_{\mathrm{f}, \mathrm{AC}}$ and a strain term $\varepsilon_{\mathrm{f}, \mathrm{C}}$ :

$$
\varepsilon_{\mathrm{f}}=\varepsilon_{\mathrm{f}, \mathrm{AC}}\left(A_{\mathrm{m}}, C_{\mathrm{m}}\right)+\varepsilon_{\mathrm{f}, \mathrm{C}}\left(C_{\mathrm{m}}\right)
$$

First, integration of Eq. (A6) with respect to $A_{\mathrm{m}}$ renders strain term $\varepsilon_{\mathrm{f}, \mathrm{AC}}$ under the condition that $C_{\mathrm{m}}$ remains constant during integration:

$$
\varepsilon_{\mathrm{f}, \mathrm{AC}}\left(A_{\mathrm{m}}, C_{\mathrm{m}}\right)=\frac{1}{2} \ln \left(\frac{A_{\mathrm{m}}}{A_{\mathrm{m}, \mathrm{ref}}}\right)+\frac{1}{4 z} \ln \left(\frac{(1+z)^{1+z}}{(1-z)^{1-z}}\right)
$$

where $A_{\mathrm{m}, \text { ref }}$ represents a reference value of midwall surface area $A_{\mathrm{m}}$. Below, the term $\varepsilon_{\mathrm{f}, \mathrm{C}}$ will be derived for a relatively simple special case of inflating a closed sphere. The thus found expression is valid for all combinations of $A_{\mathrm{m}}$ and $C_{\mathrm{m}}$. For a closed thick-walled sphere myofiber strain $\varepsilon_{\mathrm{f}}$ is known as a function of $V_{\mathrm{m}}$. Using the principle that work as generated by the myofibers equals pump work, it holds:

$$
V_{\mathrm{w}} \sigma_{\mathrm{f}} d \varepsilon_{\mathrm{f}}=\left.p_{\text {Trans }} d V_{\mathrm{m}}\right|_{\text {sphere }}
$$

Substitution of Eq. (A1) in Eq. (A9) renders for a closed sphere:

$$
\frac{d \varepsilon_{\mathrm{f}}}{d V_{\mathrm{m}}}=\left.\frac{1}{3 V_{\mathrm{w}}} \ln \left(\frac{1+z}{1-z}\right)\right|_{\text {sphere }}
$$

Then, midwall volume $V_{\mathrm{m}}$ and curvature ratio $z$ are expressed as functions of curvature $C_{\mathrm{m}}$ :

$$
V_{\mathrm{m}}=\left.\frac{4 \pi}{3 C_{\mathrm{m}}^{3}}\right|_{\text {sphere }}
$$




$$
z=\left.\frac{3 V_{\mathrm{w}} C_{\mathrm{m}}^{3}}{8 \pi}\right|_{\text {sphere }}
$$

Substitution of Eqs. (A11) and (A12) in Eq. (A10) results in a differential equation in $C_{\mathrm{m}}$ only. Integration of this differential equation with respect to $C_{\mathrm{m}}$ yields an expression of myofiber strain $\varepsilon_{\mathrm{f}}$ for a closed sphere as function of $C_{\mathrm{m}}$ (full equation given in Appendix C):

$$
\varepsilon_{\mathrm{f}}=\left.f\left(C_{\mathrm{m}}\right)\right|_{\text {sphere }}
$$

Applying Eq. (A8) to a closed sphere with curvature $C_{\mathrm{m}}$ and substituting the resulting expression as well as Eq. (A13) in Eq. (A7), results in the unknown strain term $\varepsilon_{f, C}$ (full equation given in Appendix C):

$$
\varepsilon_{\mathrm{f}, \mathrm{C}}\left(C_{\mathrm{m}}\right)=\left.\varepsilon_{\mathrm{f}}\right|_{\text {sphere }}-\left.\varepsilon_{\mathrm{f}, \mathrm{AC}}\left(A_{\mathrm{m}}, C_{\mathrm{m}}\right)\right|_{\text {sphere }}
$$

Finally, substitution of Eqs. (A8) and (A14) in Eq. (A7) yields a complicated analytical expression of natural myofiber strain $\varepsilon_{\mathrm{f}}$ in a curved wall patch (Appendix C). Mathematica 5.2 (Wolfram Research, Inc) was used to obtain a simpler fourth-order polynomial fit as specified in Eq. (15). This latter expression closely approximates the analytical derivation (relative error $<1 \%$ ).

\section{APPENDIX B: MODEL OF MYOFIBER MECHANICS}

In this appendix, the applied heuristic relation is presented that converts natural myofiber strain $\varepsilon_{\mathrm{f}}$ to Cauchy myofiber stress $\sigma_{\mathrm{f}}$. This empirical representation of sarcomere contraction simulates experiments on isolated rat cardiac muscle. ${ }^{15,64}$ Parameter values are given in Table B1.

\begin{tabular}{|c|c|c|c|}
\hline Symbol & Unit & Value & Parameter description \\
\hline$C_{\text {rest }}$ & - & 0.02 & Diastolic resting level of activation \\
\hline$L_{\mathrm{sc0}}$ & $\mu \mathrm{m}$ & 1.51 & $\begin{array}{l}\text { Contractile element length } \\
\text { with zero active stress }\end{array}$ \\
\hline$L_{\mathrm{s}, \text { ref }}$ & $\mu \mathrm{m}$ & 2.0 & $\begin{array}{l}\text { Reference sarcomere length } \\
\text { at zero strain }\end{array}$ \\
\hline$L_{\text {se,iso }}$ & $\mu \mathrm{m}$ & 0.04 & $\begin{array}{l}\text { Length of isometrically stressed } \\
\text { series elastic element }\end{array}$ \\
\hline$v_{\max }$ & $\mu \mathrm{m} \mathrm{s}^{-1}$ & 7 & $\begin{array}{l}\text { Sarcomere shortening velocity } \\
\text { with zero load }\end{array}$ \\
\hline$\tau_{\mathrm{D}}$ & $\mathrm{ms}$ & 32 & Factor scaling contraction decay time \\
\hline$\tau_{\mathrm{R}}$ & $\mathrm{ms}$ & 48 & Factor scaling contraction rise time \\
\hline$\tau_{\mathrm{sc}}$ & $\mathrm{ms}$ & 425 & Factor scaling duration of contraction \\
\hline$\sigma_{\text {act }}$ & $\mathrm{kPa}$ & 120 & Factor scaling active myofiber stress \\
\hline$\sigma_{\text {pas }}$ & $\mathrm{kPa}$ & 7 & $\begin{array}{l}\text { Factor scaling passive myofiber } \\
\text { stress }\end{array}$ \\
\hline
\end{tabular}

TABLE B1. Parameter values of the sarcomere mechanics model.
Natural myofiber strain is converted to sarcomere length $L_{\mathrm{s}}$ by

$$
L_{\mathrm{s}}=L_{\mathrm{s}, \mathrm{ref}} e^{\varepsilon_{\mathrm{f}}}
$$

The sarcomere has been modeled as a passive element in parallel with a series combination of a contractile element and series elastic element. The timedependent behavior is described by two state variables, i.e., contractile element length $L_{\mathrm{sc}}$ and mechanical activation $C$. This latter activation parameter is physiologically related to intracellular calcium concentration. The time derivative of $L_{\mathrm{sc}}$ depends linearly on length of the series elastic element $\left(L_{\mathrm{s}}-L_{\mathrm{sc}}\right)$ and equals zero for isometric contraction:

$$
\frac{d L_{\mathrm{sc}}}{d t}=\left(\frac{L_{\mathrm{s}}-L_{\mathrm{sc}}}{L_{\mathrm{se}, \text { iso }}}-1\right) v_{\max }
$$

where $v_{\max }$ represents velocity of sarcomere shortening with zero load and $L_{\mathrm{se}}$,iso length of the isometrically stressed series elastic element (Table B1). Dependence on $v_{\max }$ represents the myofiber force--velocity relation so that shortening velocity increases with applied external force.

The time derivative of $C$ is heuristically obtained, having separate terms to describe rise and decay of mechanical activation $C$ :

$$
\frac{d C}{d t}=\frac{1}{\tau_{\mathrm{R}}} \cdot C_{\mathrm{L}}\left(L_{\mathrm{sc}}\right) \cdot F_{\text {rise }}(t)+\frac{1}{\tau_{\mathrm{D}}} \cdot \frac{C_{\text {rest }}-C}{1+e^{\left(T\left(L_{\mathrm{sc}}\right)-t\right) / \tau_{\mathrm{D}}}}
$$

Parameters $\tau_{\mathrm{R}}$ and $\tau_{\mathrm{D}}$ are scaling rise and decay time, respectively (Table B1). Symbols $t$ and $C_{\text {rest }}$ represent time and diastolic resting level of activation, respectively. Functions $C_{\mathrm{L}}, F_{\text {rise }}$, and $T$ describe increase of activation with sarcomere length, rise of mechanical activation, and decrease of activation duration with decrease of sarcomere length, respectively:

$$
\begin{gathered}
C_{\mathrm{L}}\left(L_{\mathrm{sc}}\right)=\tanh \left(4.0\left(L_{\mathrm{sc}}-L_{\mathrm{sc} 0}\right)^{2}\right) \\
F_{\text {rise }}(t)=0.02 \cdot x^{3}(8-x)^{2} \mathrm{e}^{-x} \\
\text { with } x=\min \left(8, \max \left(0, t / \tau_{\mathrm{R}}\right)\right) \\
T\left(L_{\mathrm{sc}}\right)=\tau_{\mathrm{sc}}\left(0.29+0.3 L_{\mathrm{sc}}\right)
\end{gathered}
$$

where $L_{\mathrm{sc} 0}$ and $\tau_{\mathrm{sc}}$ represent contractile element length with zero load and a time factor scaling duration of contraction, respectively (Table $\mathrm{B} 1$ ).

Active myofiber stress $\sigma_{\mathrm{f} \text {,act }}$ depends on length of series elastic element, $L_{\mathrm{sc}}$, and $C$ :

$$
\sigma_{\mathrm{f}, \mathrm{act}}=\sigma_{\mathrm{act}} \cdot C \cdot\left(L_{\mathrm{sc}}-L_{\mathrm{sc} 0}\right) \cdot \frac{L_{\mathrm{s}}-L_{\mathrm{sc}}}{L_{\mathrm{se}, \text { iso }}}
$$


In order to simulate the contributions of titin as well as collagen to passive myocardial stiffness, ${ }^{69}$ passive stress $\sigma_{\mathrm{f} \text {,pas }}$ represents a soft behavior for low myofiber strain and a stiff behavior for large positive strain:

$$
\begin{aligned}
\sigma_{\mathrm{f}, \mathrm{pas}}=\sigma_{\mathrm{pas}}( & 36 \cdot \max \left(0, \varepsilon_{\mathrm{f}}-0.1\right)^{2}+0.1\left(\varepsilon_{\mathrm{f}}-0.1\right) \\
+ & \left.0.0025 \mathrm{e}^{30 \varepsilon_{\mathrm{f}}}\right)
\end{aligned}
$$

The value of $\sigma_{\text {pas }}$ has been adapted so that at maximum sarcomere length with exercise, passive myofiber stress assumes the maximum value (Table 1: Set point values for adaptation). Total Cauchy myofiber stress $\sigma_{\mathrm{f}}$ is the sum of active and passive stress:

$$
\sigma_{\mathrm{f}}\left(\varepsilon_{\mathrm{f}}\right)=\sigma_{\mathrm{f}, \mathrm{pas}}+\sigma_{\mathrm{f}, \mathrm{act}}
$$

The latter result has been substituted in Eq. (16).

\section{APPENDIX C: POLYNOMIAL SERIES APPROXIMATIONS}

The polynomial series approximation given by Eq. (15) is obtained from the analytical solution expressing natural myofiber strain in terms of $A_{\mathrm{m}}$ and $C_{\mathrm{m}}$ (Eq. A7) by applying the following steps:

1. Expression of myofiber strain $\varepsilon_{\mathrm{f}}$ for a closed sphere (use $A_{\mathrm{m}}=4 \pi / C_{\mathrm{m}}^{2}$ ) as function of $C_{\mathrm{m}}$ (Eq. A13) yields:

$$
\begin{gathered}
\left.\varepsilon_{\mathrm{f}}\right|_{\text {sphere }}=-\ln \left(C_{\mathrm{m}}\right)+\frac{\ln (8 \pi)}{3}+\frac{G(x)}{6} \\
G(x)=\frac{1}{x} \cdot \ln \left(\frac{(1+x)^{1+x}}{(1-x)^{1-x}}\right) \\
x=\left.z\right|_{\text {sphere }}=\frac{3 C_{m}^{3} V_{w}}{8 \pi}
\end{gathered}
$$

2. Applying Eq. (A8) to a closed sphere (use $\left.A_{\mathrm{m}}=4 \pi / C_{\mathrm{m}}^{2}\right)$ yields:

$$
\left.\varepsilon_{\mathrm{f}, \mathrm{AC}}\right|_{\text {sphere }}=-\ln \left(C_{\mathrm{m}}\right)+\frac{1}{2} \cdot \ln \left(\frac{4 \pi}{A_{\mathrm{m}, \mathrm{ref}}}\right)+\frac{G(x)}{4}
$$

Substitution of Eqs. (C1a) and (C2) in Eq. (A14) yields for strain term $\varepsilon_{\mathrm{f}, \mathrm{C}}$ :

$\varepsilon_{\mathrm{f}, \mathrm{C}}=\frac{\ln (8 \pi)}{3}-\frac{1}{2} \cdot \ln \left(\frac{4 \pi}{A_{\mathrm{m}, \mathrm{ref}}}\right)-\frac{G(x)}{12}$

The thus found expression for $\varepsilon_{\mathrm{f}, \mathrm{C}}$, which holds for the special case of inflating a sphere, is valid for all combinations of $A_{\mathrm{m}}$ and $C_{\mathrm{m}}$.
3. Substitution of Eqs. (C3) and (A8) in Eq. (A7) yields the general analytical solution of natural myofiber strain $\varepsilon_{\mathrm{f}}$ in a curved wall patch:

$$
\begin{aligned}
\varepsilon_{\mathrm{f}}= & \frac{\ln (8 \pi)}{3}-\frac{1}{2} \cdot \ln \left(\frac{4 \pi}{A_{\mathrm{m}, \mathrm{ref}}}\right)+\frac{1}{2} \cdot \ln \left(\frac{A_{\mathrm{m}}}{A_{\mathrm{m}, \text { ref }}}\right) \\
& -\frac{G(x)}{12}+\frac{G(z)}{4}
\end{aligned}
$$

4. In order to facilitate series approximation, $z=$ $K / A$ and $x=K^{3}$ are substituted in Eq. (C4):

$$
\begin{aligned}
\varepsilon_{\mathrm{f}}= & \frac{\ln (8 \pi)}{3}-\frac{1}{2} \cdot \ln \left(\frac{4 \pi}{A_{\mathrm{m}, \mathrm{ref}}}\right)+\frac{1}{2} \cdot \ln \left(\frac{A_{\mathrm{m}}}{A_{\mathrm{m}, \mathrm{ref}}}\right) \\
& -\frac{1}{12 K^{3}} \cdot \ln \left(\frac{\left(1+K^{3}\right)^{1+K^{3}}}{\left(1-K^{3}\right)^{1-K^{3}}}\right) \\
& +\frac{A}{4 K} \cdot \ln \left(\frac{\left(1+\frac{K}{A}\right)^{1+\frac{K}{A}}}{\left(1-\frac{K}{A}\right)^{1-\frac{K}{A}}}\right)
\end{aligned}
$$

with $A$ and $K$ representing normalized area and curvature, respectively:

$$
\begin{gathered}
A=\frac{A_{\mathrm{m}}}{\sqrt[3]{9 \pi V_{\mathrm{w}}^{2}}} \\
K=\frac{C_{\mathrm{m}}}{2} \sqrt[3]{\frac{3 V_{\mathrm{w}}}{\pi}}
\end{gathered}
$$

5. The first two terms of Eq. (C5a) are constant strain offsets and are therefore eliminated from the strain relation, which describes the transition from a reference to a new state with state variables $A_{\mathrm{m}}$ and $C_{\mathrm{m}}$. Taylor series approximation of this simplified version of Eq. (C5a) yields:

$$
\varepsilon_{\mathrm{f}}=\frac{1}{2} \cdot \ln \left(\frac{A_{\mathrm{m}}}{A_{\mathrm{m}, \mathrm{ref}}}\right)-\frac{K^{2}}{12 A^{2}}-\frac{K^{4}}{40 A^{4}}+\mathrm{O}\left(K^{6}\right)
$$

where $\mathrm{O}\left(K^{6}\right)$ represents the higher order terms.

6. It is used that $z=K / A$. Furthermore, the fourthorder polynomial approximation of natural myofiber strain in a curved wall patch (Eq. 15) is obtained after slight adjustment of the fourthorder term of Eq. (C6) for best accuracy within the whole range of operation $(|z|<0.8)$.

The polynomial series approximation given by Eq. (16) is obtained from the analytical solution expressing representative midwall tension in terms of $A_{\mathrm{m}}, C_{\mathrm{m}}$, and $\sigma_{\mathrm{f}}$ (Eq. A4) by applying the following steps:

1. Substitution of $z=3 C_{\mathrm{m}} V_{\mathrm{w}} / 2 A_{\mathrm{m}}$ in Eq. (A4) yields:

$$
\frac{T_{\mathrm{m}}}{\sigma_{\mathrm{f}}}=\frac{1}{6 C_{\mathrm{m}}} \ln \left(\frac{2 A_{\mathrm{m}}+3 C_{\mathrm{m}} V_{\mathrm{w}}}{2 A_{\mathrm{m}}-3 C_{\mathrm{m}} V_{\mathrm{w}}}\right)
$$


2. Similar to what has been done to obtain Eq. (C5a), $A$ and $K$ are substituted in Eq. (C7) to facilitate series approximation:

$$
\frac{T_{\mathrm{m}}}{\sigma_{\mathrm{f}}}=\frac{L}{6 K} \ln \left(\frac{1+\frac{K}{A}}{1-\frac{K}{A}}\right)
$$

with

$$
L=\frac{1}{2} \sqrt[3]{\frac{3 V_{\mathrm{w}}}{\pi}}
$$

3. Taylor series approximation of Eq. (C8a) yields:

$$
\frac{T_{\mathrm{m}}}{\sigma_{f}}=\frac{L}{3 A}+\frac{L K^{2}}{9 A^{3}}+\frac{L K^{4}}{15 A^{5}}+\mathrm{O}\left(K^{6}\right)
$$

where $\mathrm{O}\left(K^{6}\right)$ represents the higher order terms, which are neglected in the TriSeg model.

4. Finally, the fourth-order polynomial approximation of representative midwall tension (Eq. 16), is obtained by rearranging Eq. (C9) and using $z=K / A$.

\section{ACKNOWLEDGMENTS}

We gratefully acknowledge the financial support of Actelion Pharmaceuticals Nederland B.V. (Woerden, The Netherlands) and the Netherlands Heart Foundation Grant 2007B203.

\section{OPEN ACCESS}

This article is distributed under the terms of the Creative Commons Attribution Noncommercial License which permits any noncommercial use, distribution, and reproduction in any medium, provided the original author(s) and source are credited.

\section{REFERENCES}

\footnotetext{
${ }^{1}$ Arts, T., P. Bovendeerd, T. Delhaas, and F. Prinzen. Modeling the relation between cardiac pump function and myofiber mechanics. J. Biomech. 36:731-736, 2003.

${ }^{2}$ Arts, T., P. H. Bovendeerd, F. W. Prinzen, and R. S. Reneman. Relation between left ventricular cavity pressure and volume and systolic fiber stress and strain in the wall. Biophys. J. 59:93-102, 1991.

${ }^{3}$ Arts, T., T. Delhaas, P. Bovendeerd, X. Verbeek, and F. W. Prinzen. Adaptation to mechanical load determines shape and properties of heart and circulation: the CircAdapt model. Am. J. Physiol. Heart Circ. Physiol. 288:H1943-H1954, 2005.

${ }^{4}$ Arts, T., F. W. Prinzen, L. H. Snoeckx, J. M. Rijcken, and R. S. Reneman. Adaptation of cardiac structure by
}

mechanical feedback in the environment of the cell: a model study. Biophys. J. 66:953-961, 1994.

${ }^{5}$ Baker, A. E., R. Dani, E. R. Smith, J. V. Tyberg, and I. Belenkie. Quantitative assessment of independent contributions of pericardium and septum to direct ventricular interaction. Am. J. Physiol. 275:H476-H483, 1998.

${ }^{6}$ Belenkie, I., R. Dani, E. R. Smith, and J. V. Tyberg. The importance of pericardial constraint in experimental pulmonary embolism and volume loading. Am. Heart J. 123:733-742, 1992.

${ }^{7}$ Beyar, R., S. J. Dong, E. R. Smith, I. Belenkie, and J. V. Tyberg. Ventricular interaction and septal deformation: a model compared with experimental data. Am. J. Physiol. 265:H2044-H2056, 1993.

${ }^{8}$ Blanchard, D. G., and H. C. Dittrich. Pericardial adaptation in severe chronic pulmonary hypertension. An intraoperative transesophageal echocardiographic study. Circulation 85:1414-1422, 1992.

${ }^{9}$ Bossone, E., E. Avelar, D. S. Bach, B. Gillespie, M. Rubenfire, and W. F. Armstrong. Diagnostic value of resting tricuspid regurgitation velocity and right ventricular ejection flow parameters for the detection of exercise induced pulmonary arterial hypertension. Int. J. Card. Imaging 16:429-436, 2000.

${ }^{10}$ Brinker, J. A., J. L. Weiss, D. L. Lappe, J. L. Rabson, W. R. Summer, S. Permutt, and M. L. Weisfeldt. Leftward septal displacement during right ventricular loading in man. Circulation 61:626-633, 1980.

${ }^{11}$ Calvin, J. E. Optimal right ventricular filling pressures and the role of pericardial constraint in right ventricular infarction in dogs. Circulation 84:852-861, 1991.

${ }^{12}$ Chemla, D., V. Castelain, P. Herve, Y. Lecarpentier, and S. Brimioulle. Haemodynamic evaluation of pulmonary hypertension. Eur. Respir. J. 20:1314-1331, 2002.

${ }^{13}$ Chung, D. C., S. C. Niranjan, J. W. Clark, Jr., A. Bidani, W. E. Johnston, J. B. Zwischenberger, and D. L. Traber. A dynamic model of ventricular interaction and pericardial influence. Am. J. Physiol. 272:H2942-H2962, 1997.

${ }^{14}$ Dabestani, A., G. Mahan, J. M. Gardin, K. Takenaka, C. Burn, A. Allfie, and W. L. Henry. Evaluation of pulmonary artery pressure and resistance by pulsed Doppler echocardiography. Am. J. Cardiol. 59:662-668, 1987.

${ }^{15} \mathrm{de}$ Tombe, P. P., and H. E. ter Keurs. Force and velocity of sarcomere shortening in trabeculae from rat heart. Effects of temperature. Circ. Res. 66:1239-1254, 1990.

${ }^{16}$ Debnath, L. Nonlinear Partial Differential Equations for Scientists and Engineers (2nd ed.). Boston: Birkhäuser, p. 737, 2005.

${ }^{17}$ Dellegrottaglie, S., J. Sanz, M. Poon, J. F. Viles-Gonzalez, R. Sulica, M. Goyenechea, F. Macaluso, V. Fuster, and S. Rajagopalan. Pulmonary hypertension: accuracy of detection with left ventricular septal-to-free wall curvature ratio measured at cardiac MR. Radiology 243:63-69, 2007.

${ }^{18}$ Dittrich, H. C., H. A. McCann, and D. G. Blanchard. Cardiac structure and function in chronic thromboembolic pulmonary hypertension. Am. J. Card. Imaging 8:18-27, 1994.

${ }^{19}$ Doherty, 3rd, N. E., N. Fujita, G. R. Caputo, and C. B. Higgins. Measurement of right ventricular mass in normal and dilated cardiomyopathic ventricles using cine magnetic resonance imaging. Am. J. Cardiol. 69:1223-1228, 1992.

${ }^{20}$ Dong, S. J., A. P. Crawley, J. H. MacGregor, Y. F. Petrank, D. W. Bergman, I. Belenkie, E. R. Smith, J. V. Tyberg, and R. Beyar. Regional left ventricular systolic function in relation to the cavity geometry in patients with 
chronic right ventricular pressure overload. A threedimensional tagged magnetic resonance imaging study. Circulation 91:2359-2370, 1995.

${ }^{21}$ Dong, S. J., E. R. Smith, and J. V. Tyberg. Changes in the radius of curvature of the ventricular septum at end diastole during pulmonary arterial and aortic constrictions in the dog. Circulation 86:1280-1290, 1992.

${ }^{22}$ Donker, D. W., P. G. Volders, T. Arts, B. C. Bekkers, L. Hofstra, R. L. Spatjens, J. D. Beekman, M. Borgers, H. J. Crijns, and M. A. Vos. End-diastolic myofiber stress and ejection strain increase with ventricular volume overload - serial in vivo analyses in dogs with complete atrioventricular block. Basic Res. Cardiol. 100:372-382, 2005.

${ }^{23}$ Faber, M. J., M. Dalinghaus, I. M. Lankhuizen, P. Steendijk, W. C. Hop, R. G. Schoemaker, D. J. Duncker, J. M. Lamers, and W. A. Helbing. Right and left ventricular function after chronic pulmonary artery banding in rats assessed with biventricular pressure-volume loops. Am. J. Physiol. Heart Circ. Physiol. 291:H1580-H1586, 2006.

${ }^{24}$ Freeman, G. L., and M. M. LeWinter. Pericardial adaptations during chronic cardiac dilation in dogs. Circ. Res. 54:294-300, 1984.

${ }^{25}$ Freeman, G. L., and M. M. LeWinter. Role of parietal pericardium in acute, severe mitral regurgitation in dogs. Am. J. Cardiol. 54:217-219, 1984.

${ }^{26}$ Fujii, J., Y. Yazaki, H. Sawada, T. Aizawa, H. Watanabe, and K. Kato. Noninvasive assessment of left and right ventricular filling in myocardial infarction with a twodimensional Doppler echocardiographic method. J. Am. Coll. Cardiol. 5:1155-1160, 1985.

${ }^{27}$ Gardin, J. M., C. S. Burn, W. J. Childs, and W. L. Henry. Evaluation of blood flow velocity in the ascending aorta and main pulmonary artery of normal subjects by Doppler echocardiography. Am. Heart J. 107:310-319, 1984.

${ }^{28}$ Hajduczok, Z. D., R. M. Weiss, W. Stanford, and M. L. Marcus. Determination of right ventricular mass in humans and dogs with ultrafast cardiac computed tomography. Circulation 82:202-212, 1990.

${ }^{29}$ Hirschfeld, S., R. Meyer, D. C. Schwartz, J. Korfhagen, and S. Kaplan. Measurement of right and left ventricular systolic time intervals by echocardiography. Circulation 51:304-309, 1975.

${ }^{30}$ Horan, L. G., N. C. Flowers, and C. J. Havelda. Relation between right ventricular mass and cavity size: an analysis of 1500 human hearts. Circulation 64:135-138, 1981.

${ }^{31}$ Hunter, P. J., A. D. McCulloch, and H. E. ter Keurs. Modelling the mechanical properties of cardiac muscle. Prog. Biophys. Mol. Biol. 69:289-331, 1998.

${ }^{32} \mathrm{Kaul}, \mathrm{S}$. The interventricular septum in health and disease. Am. Heart J. 112:568-581, 1986.

${ }^{33}$ Kerckhoffs, R. C., J. Lumens, K. Vernooy, J. H. Omens, L. J. Mulligan, T. Delhaas, T. Arts, A. D. McCulloch, and F. W. Prinzen. Cardiac resynchronization: insight from experimental and computational models. Prog. Biophys. Mol. Biol. 97:543-561, 2008.

${ }^{34}$ Kerckhoffs, R. C., M. L. Neal, Q. Gu, J. B. Bassingthwaighte, J. H. Omens, and A. D. McCulloch. Coupling of a 3D finite element model of cardiac ventricular mechanics to lumped systems models of the systemic and pulmonic circulation. Ann. Biomed. Eng. 35:1-18, 2007.

${ }^{35}$ King, M. E., H. Braun, A. Goldblatt, R. Liberthson, and A. E. Weyman. Interventricular septal configuration as a predictor of right ventricular systolic hypertension in children: a cross-sectional echocardiographic study. Circulation 68:68-75, 1983 .
${ }^{36}$ Kingma, I., J. V. Tyberg, and E. R. Smith. Effects of diastolic transseptal pressure gradient on ventricular septal position and motion. Circulation 68:1304-1314, 1983.

${ }^{37}$ Little, W. C., R. C. Reeves, J. Arciniegas, R. E. Katholi, and E. W. Rogers. Mechanism of abnormal interventricular septal motion during delayed left ventricular activation. Circulation 65:1486-1491, 1982.

${ }^{38}$ Louie, E. K., S. S. Lin, S. I. Reynertson, B. H. Brundage, S. Levitsky, and S. Rich. Pressure and volume loading of the right ventricle have opposite effects on left ventricular ejection fraction. Circulation 92:819-824, 1995.

${ }^{39}$ Louie, E. K., S. Rich, S. Levitsky, and B. H. Brundage. Doppler echocardiographic demonstration of the differential effects of right ventricular pressure and volume overload on left ventricular geometry and filling. J. Am. Coll. Cardiol. 19:84-90, 1992.

${ }^{40}$ Lumens, J., T. Delhaas, B. Kirn, and T. Arts. Modeling ventricular interaction: a multiscale approach from sarcomere mechanics to cardiovascular system hemodynamics. Pac. Symp. Biocomput. 378-389, 2008.

${ }^{41}$ Luo, C., D. L. Ware, J. B. Zwischenberger, and J. W. Clark, Jr. Using a human cardiopulmonary model to study and predict normal and diseased ventricular mechanics, septal interaction, and atrio-ventricular blood flow patterns. Cardiovasc. Eng. 7:17-31, 2007.

${ }^{42}$ Mangano, D. T., D. C. Van Dyke, R. F. Hickey, and R. J. Ellis. Significance of the pericardium in human subjects: effects on left ventricular volume, pressure and ejection. J. Am. Coll. Cardiol. 6:290-295, 1985.

${ }^{43}$ Maughan, W. L., K. Sunagawa, and K. Sagawa. Ventricular systolic interdependence: volume elastance model in isolated canine hearts. Am. J. Physiol. 253:H1381-H1390, 1987.

${ }^{44}$ Minami, S., T. Onodera, F. Okazaki, H. Miyazaki, S. Ohsawa, and S. Mochizuki. Myocyte morphological characteristics differ between the phases of pulmonary hypertension-induced ventricular hypertrophy and failure. Int. Heart J. 47:629-637, 2006.

${ }^{45}$ Miyamoto, S., N. Nagaya, T. Satoh, S. Kyotani, F. Sakamaki, M. Fujita, N. Nakanishi, and K. Miyatake. Clinical correlates and prognostic significance of six-minute walk test in patients with primary pulmonary hypertension. Comparison with cardiopulmonary exercise testing. Am. J. Respir. Crit. Care Med. 161:487-492, 2000.

${ }^{46}$ Morris-Thurgood, J. A., and M. P. Frenneaux. Diastolic ventricular interaction and ventricular diastolic filling. Heart Fail. Rev. 5:307-323, 2000.

${ }^{47}$ Nash, M. P., and P. J. Hunter. Computational mechanics of the heart: from tissue structure to ventricular function. J. Elast. 61:113-141, 2000.

${ }^{48}$ Olansen, J. B., J. W. Clark, D. Khoury, F. Ghorbel, and A. Bidani. A closed-loop model of the canine cardiovascular system that includes ventricular interaction. Comput. Biomed. Res. 33:260-295, 2000.

${ }^{49}$ Olivetti, G., R. Ricci, C. Lagrasta, E. Maniga, E. H. Sonnenblick, and P. Anversa. Cellular basis of wall remodeling in long-term pressure overload-induced right ventricular hypertrophy in rats. Circ. Res. 63:648-657, 1988.

${ }^{50}$ Olsen, C. O., G. S. Tyson, G. W. Maier, J. A. Spratt, J. W. Davis, and J. S. Rankin. Dynamic ventricular interaction in the conscious dog. Circ. Res. 52:85-104, 1983.

${ }^{51}$ Reisner, S. A., Z. Azzam, M. Halmann, D. Rinkevich, S. Sideman, W. Markiewicz, and R. Beyar. Septal/free wall curvature ratio: a noninvasive index of pulmonary arterial pressure. J. Am. Soc. Echocardiogr. 7:27-35, 1994. 
${ }^{52}$ Roeleveld, R. J., J. T. Marcus, T. J. Faes, T. J. Gan, A. Boonstra, P. E. Postmus, and A. Vonk-Noordegraaf. Interventricular septal configuration at $\mathrm{mr}$ imaging and pulmonary arterial pressure in pulmonary hypertension. Radiology 234:710-717, 2005.

${ }^{53}$ Santamore, W. P., and D. Burkhoff. Hemodynamic consequences of ventricular interaction as assessed by model analysis. Am. J. Physiol. 260:H146-H157, 1991.

${ }^{54}$ Sechtem, U., P. W. Pflugfelder, R. G. Gould, M. M. Cassidy, and C. B. Higgins. Measurement of right and left ventricular volumes in healthy individuals with cine MR imaging. Radiology 163:697-702, 1987.

${ }^{55}$ Slinker, B. K., and S. A. Glantz. End-systolic and enddiastolic ventricular interaction. Am. J. Physiol. 251: H1062-H1075, 1986.

${ }^{56}$ Smith, B. W., J. G. Chase, G. M. Shaw, and R. I. Nokes. Simulating transient ventricular interaction using a minimal cardiovascular system model. Physiol. Meas. 27:165179, 2006.

${ }^{57}$ Stolzmann, P., H. Scheffel, S. Leschka, T. Schertler, T. Frauenfelder, P. A. Kaufmann, B. Marincek, and H. Alkadhi. Reference values for quantitative left ventricular and left atrial measurements in cardiac computed tomography. Eur. Radiol. 18:1625-1634, 2008.

${ }^{58}$ Streeter Jr., D. D. Gross morphology and fiber geometry of the heart. In: Handbook of Physiology, edited by R. M. Berne, N. Sperelakis, and S. R. Geiger. Bethesda, MD: American Physiological Society, 1979, pp. 61-112.

${ }^{59}$ Suga, H., T. Hayashi, and M. Shirahata. Ventricular systolic pressure-volume area as predictor of cardiac oxygen consumption. Am. J. Physiol. 240:H39-H44, 1981.

${ }^{60}$ Sun, Y., M. Beshara, R. J. Lucariello, and S. A. Chiaramida. A comprehensive model for right-left heart interaction under the influence of pericardium and baroreflex. Am. J. Physiol. 272:H1499-H1515, 1997.

${ }^{61}$ Taher, M. F., W. P. Santamore, and D. K. Bogen. Ventricular interaction is described by three coupling coefficients. Am. J. Physiol. 266:H228-H234, 1994.
${ }^{62}$ Tei, C., K. S. Dujardin, D. O. Hodge, K. R. Bailey, M. D. McGoon, A. J. Tajik, and S. B. Seward. Doppler echocardiographic index for assessment of global right ventricular function. J. Am. Soc. Echocardiogr. 9:838-847, 1996.

${ }^{63}$ Tei, C., K. S. Dujardin, D. O. Hodge, R. A. Kyle, A. J. Tajik, and J. B. Seward. Doppler index combining systolic and diastolic myocardial performance: clinical value in cardiac amyloidosis. J. Am. Coll. Cardiol. 28:658-664, 1996.

${ }^{64}$ ter Keurs, H. E., W. H. Rijnsburger, R. van Heuningen, and M. J. Nagelsmit. Tension development and sarcomere length in rat cardiac trabeculae. Evidence of lengthdependent activation. Circ. Res. 46:703-714, 1980.

${ }^{65}$ Thistlethwaite, P. A., and S. W. Jamieson. Tricuspid valvular disease in the patient with chronic pulmonary thromboembolic disease. Curr. Opin. Cardiol. 18:111-116, 2003.

${ }^{66}$ Usyk, T. P., I. J. Le Grice, and A. D. McCulloch. Computational model of three-dimensional cardiac electromechanics. Comput. Vis. Sci. 4:249-257, 2002.

${ }^{67}$ Watkins, M. W., and M. M. LeWinter. Physiologic role of the normal pericardium. Annu. Rev. Med. 44:171-180, 1993.

${ }^{68}$ Weber, K. T., J. S. Janicki, S. Shroff, and A. P. Fishman. Contractile mechanics and interaction of the right and left ventricles. Am. J. Cardiol. 47:686-695, 1981.

${ }^{69}$ Wu, Y., O. Cazorla, D. Labeit, S. Labeit, and H. Granzier. Changes in titin and collagen underlie diastolic stiffness diversity of cardiac muscle. J. Mol. Cell. Cardiol. 32:21512162, 2000.

${ }^{70}$ Yeo, T. C., K. S. Dujardin, C. Tei, D. W. Mahoney, M. D. McGoon, and J. B. Seward. Value of a Doppler-derived index combining systolic and diastolic time intervals in predicting outcome in primary pulmonary hypertension. Am. J. Cardiol. 81:1157-1161, 1998.

${ }^{71}$ Yu, C. M., J. E. Sanderson, S. Chan, L. Yeung, Y. T. Hung, and K. S. Woo. Right ventricular diastolic dysfunction in heart failure. Circulation 93:1509-1514, 1996. 